\title{
A fully discrete energy stable scheme for a phase filed moving contact line model with variable densities and viscosities
}

\author{
Guangpu Zhua, Huangxin Chen ${ }^{\mathrm{b}}$, Shuyu Sun ${ }^{\mathrm{c}, *}$ and Jun Yao ${ }^{\mathrm{a}, *}$ \\ ${ }^{a}$ Research Center of Multiphase Flow in Porous Media, School of Petroleum Engineering, China University of Petroleum (East \\ China), Qingdao 266580, China \\ ${ }^{\mathrm{b}}$ School of Mathematical Sciences and Fujian Provincial Key Laboratory on Mathematical Modeling and High Performance \\ Scientific Computing, Xiamen University, Xiamen 361005, China \\ ${ }^{\circ}$ Computational Transport Phenomena Laboratory, Division of Physical Science and Engineering, King Abdullah University of \\ Science and Technology, Thuwal 23955-6900, Kingdom of Saudi Arabia
}

\begin{abstract}
In this paper, a fully discrete scheme which considers temporal and spatial discretizations is presented for the coupled Cahn-Hilliard equation in conserved form with the dynamic contact line condition and the Navier-Stokes equation with the generalized Navier boundary condition. Variable densities and viscosities are incorporated in this model. A rigorous proof of energy stability is provided for the fully discrete scheme based on a semi-implicit temporal discretization and a finite difference method on the staggered grids for the spatial discretization. A splitting method based on the pressure stabilization is implemented to solve the Navier-Stokes equation, while the stabilization approach is also used for the Cahn-Hilliard equation. Numerical results in both 2-D and 3-D demonstrate the accuracy, efficiency and decaying property of discrete energy of the proposed scheme.
\end{abstract}

\section{Introduction}

The moving contact line problem, which occurs when the interface of two immiscible fluid components touches the solid wall, is a classical problem that appears in many industrial applications. For instance, in petroleum industry, the wettability of rock affects the efficiency of water flooding on the residual oil, and thus it determines the oil recovery [1]. Handling boundary conditions on the slip boundary is always a challenging work. It is well known that the classical Navier-Stokes equation with the no-slip boundary condition is not applicable for the two-phase flow system because a non-physical singularity will occur in the vicinity of the moving contact line $[2,3]$. A series of methods have been used to understand the hydrodynamic behavior near the moving contact line, including molecular dynamics (MD) simulations [4, 5], the sharp interface models [6] and the phase filed model. We consider the phase filed model in this work. The phase field approach treats the interface as a thin and continuous layer, and a phase variable is introduced to distinguish the two immiscible phases [7-9]. The phase filed model has been widely used in the simulations of two immiscible fluid components by coupling the four-order Cahn-Hilliard equation with Navier-Stokes equation via convection and stress terms. Numerous numerical schemes [10-16], which satisfy the corresponding discrete energy law, have been proposed to improve the efficiency and stability of the phase filed model.

MD studies had proven that there exists a relative slipping between the fluid and wall.

Keywords: Phase-field; Navier-Stokes; Cahn-Hilliard; Moving contact line; Fully discrete scheme; Energy stability. *Corresponding author.

E-mail addresses: b16020069@s.upc.edu.cn (G. Zhu), chx@xmu.edu.cn (H. Chen), shuyu.sun@kaust.edu.sa (S. Sun), rcogfr_upc@126.com (J. Yao). 
Further, Qian et al. [4, 5, 17] found that the amount of slipping is proportional to the sum of tangential viscous stress and the uncompensated Young stress. They proposed a continuum governing system of immiscible flow dynamics [17], which consists of the Cahn-Hilliard equation with the dynamic contact line condition and the Navier-Stokes equation with the generalized Navier boundary condition (GNBC). The numerical results based on the continuum governing system are consistent with the results from MD simulations [4], which demonstrates that the continuum hydrodynamic model with the GNBC can describe the fluid behavior near the moving contact line accurately. Developing an efficient numerical scheme to solve such a complex dynamical system, which satisfies a thermodynamically consistent energy dissipation law, is quite a challenging topic. Recently, several attempts had been made in the literature to improve the efficiency and stability of solving the coupled Cahn-Hilliard and Navier-Stokes equations with the GNBC.

In [18], the authors proposed a least-square finite element method with a temporal discretization by the operator-splitting to overcome the difficulties associated with the boundary conditions and nonlinearity. Gao and Wang $[19,20]$ developed an efficient numerical scheme for a phase field moving contact line model with variable densities and viscosities. In [19, 20], the temporal scheme was energy stable under certain conditions, and a finite difference method on the semi-staggered grids was used to discrete the nonlinear coupled system in space. However, a rigorous proof of the energy stability of the fully discrete scheme was not provided. In addition, the schemes based on the pressure correction method and the pressure stabilization method were compared in their work. They concluded that the results calculated by the two projection schemes have the same accuracy and the former scheme is computationally much more expensive than the latter one due to the cost of solving an elliptic equation with variable coefficients. With some subtle explicit-implicit treatment of the nonlinear terms, Shen et al. [14] developed a linear decoupled energy stable scheme for the matched density system with the static contact line condition and a linear coupled scheme for the matched density system with the dynamic contact line condition. A spectral-Galerkin spatial discretization method was implemented to solve the system. Aland and Chen [21] constructed a linear coupled scheme for the matched density moving contact line problem, and they proved the unconditional energy stability for the proposed temporal discretization as well as for a fully discrete finite element scheme. Furthermore, Yu and Yang [22] proposed a nonlinear energy stable scheme for a non-matched density system with the dynamic contact line condition. The spatial discretization was completed by a spectral-Galerkin method. In addition, Yang et al. [23, 24] developed the decoupled energy stable scheme for the hydrodynamic Allen-Cahn phase filed moving contact line model and a finite element method spatial discretization method was implemented to verify the accuracy and efficiency.

The widely used finite difference method on the staggered grids, i.e., also known as the MAC method, has been widely used in engineering applications (cf. [25-29] and the references therein). It has been one of the simplest and effective numerical schemes to solve the Navier-Stokes equation. To the best of the author's knowledge, a fully discrete scheme for the coupled Cahn-Hilliard equation with dynamic contact line condition and Navier-Stokes equation with the GNBC based on the finite difference method on the staggered grids and its energy stability estimate have never been investigated in the literature. In this paper, we consider the energy stability estimate for the fully discrete scheme which considers the temporal and spatial discretizations for the coupled Cahn-Hilliard equation with the dynamic contact line condition and 
the Navier-Stokes equation with the GNBC. The semi-implicit temporal discretization proposed in [22] is employed for the temporal discretization. For the spatial discretization, a finite difference method on the staggered grids is implemented to solve the coupled system. A rigorous proof of energy stability for the fully discrete scheme is provided. Several 2-D and 3-D numerical experiments are conducted to validate the accuracy, efficiency and energy stability of the proposed scheme.

The rest of paper is organized as follows. The coupled Cahn-Hilliard and Navier-Stokes system with the moving contact line are introduced in Section 2, and variable densities and viscosities are also incorporated in the governing system. In Section 3, the fully temporal and spatial discretization are presented and the corresponding energy stability estimate are given. Several numerical examples are investigated in Section 4 and the paper is finally concluded in Section 5 .

\section{The governing equations}

The moving contact line model with variable densities and viscosities can be described by the following coupled Cahn-Hilliard equation with the dynamic contact line condition and the Navier-Stokes equation with the GNBC [22].

$$
\left\{\begin{array}{l}
\frac{\partial \phi}{\partial \mathrm{t}}+\nabla \cdot(\mathbf{u} \phi)-M \Delta w=0, \\
w+\lambda(\varepsilon \Delta \phi-f(\phi))=0, \\
\rho\left(\frac{\partial \mathbf{u}}{\partial t}+(\mathbf{u} \cdot \nabla) \mathbf{u}\right)+\mathbf{J} \cdot \nabla \mathbf{u}-\nabla \cdot \eta \nabla \mathbf{u}+\nabla p+\phi \nabla w=0, \\
\nabla \cdot \mathbf{u}=0
\end{array}\right.
$$

and

$$
f(\phi)=F^{\prime}(\phi)=\frac{\phi^{3}-\phi}{\varepsilon}, \mathbf{J}=\frac{\rho_{2}-\rho_{1}}{2} M \nabla w,
$$

where $\phi$ is the phase variable ( $\phi= \pm 1$ in this study), $M$ is the mobility parameter, $w$ is the chemical potential, $\lambda$ denotes the rescaled characteristic strength of phase mixing energy, $\varepsilon$ is a parameter proportional to the interface thickness [30], $\mathbf{u}$ is the flow velocity, $p$ is the pressure. Here $F(\phi)$ is the Ginzburg-Landau potential, and it can be written as $\left(\phi^{2}-1\right)^{2} /(4 \varepsilon)$.

Note that the density and viscosity in the above equations are defined as as follows:

$$
\begin{gathered}
\rho=\frac{\rho_{1}-\rho_{2}}{2} \phi+\frac{\rho_{1}+\rho_{2}}{2}, \\
\eta=\frac{\eta_{1}-\eta_{2}}{2} \phi+\frac{\eta_{1}+\eta_{2}}{2} .
\end{gathered}
$$

Assuming the phase interface never touches the solid wall, the following boundary conditions can be easily implemented,

$$
\left\{\begin{array}{l}
\left.(\mathbf{n} \cdot \nabla \phi)\right|_{\Gamma}=\left.(\mathbf{n} \cdot \nabla w)\right|_{\Gamma}=0 \\
\left.\mathbf{u} \cdot \mathbf{n}\right|_{\Gamma}=0
\end{array}\right.
$$

When the phase interface touches the solid wall, the GNBC should be introduced to deal with the moving contact line problem, the velocity along the solid wall can be evaluated as

$$
\left\{\begin{array}{l}
\left.\left(\eta \beta\left(\mathbf{u}_{\tau}-\mathbf{u}_{\mathbf{w}}\right)+\eta\left(\partial_{\mathbf{n}} \mathbf{u}_{\tau}+\partial_{\tau} \mathbf{u}_{\mathbf{n}}\right)-\lambda L \nabla_{\tau} \phi\right)\right|_{\Gamma}=0, \\
\left.\mathbf{u} \cdot \mathbf{n}\right|_{\Gamma}=0 .
\end{array}\right.
$$


In addition, the dynamic boundary conditions for the phase field variable are imposed as

$$
\left\{\begin{array}{l}
\left.\left(\frac{\partial \phi}{\partial t}+\mathbf{u}_{\tau} \cdot \nabla_{\tau} \phi+\gamma L(\phi)\right)\right|_{\Gamma}=0, \\
\partial_{\mathbf{n}} w=0
\end{array}\right.
$$

where $\mathbf{u}_{\mathbf{w}}$ is the wall velocity, $\mathbf{u}_{\boldsymbol{\tau}}$ and $\mathbf{u}_{\mathbf{n}}$ are the velocities along the tangential and normal direction of the slip boundary respectively, $\gamma$ is the boundary relaxation parameter, $\beta$ is the ratio of domain length to the slip length. The function $L(\phi)$ is defined as

$$
L=\varepsilon \partial_{n} \phi+g^{\prime}(\phi),
$$

and the surface energy $g(\phi)$ can be written as

$$
g(\phi)=-\frac{\sqrt{2}}{3} \cos \theta_{s} \sin \left(\frac{\pi}{2} \phi\right),
$$

where $\theta_{s}$ is the static contact angle.

\section{The numerical implementation}

\subsection{Temporal discretization}

In this work, the Ginzburg-Landau potential $F(\phi)$ is handled by the stabilization method, which can provide a linear discretization for $f(\phi)$. The unconditional stability of a stabilization method requires the second derivative of $F(\phi)$ to be bounded. Nevertheless, this is not satisfied by $F(\phi)$. A truncated potential [31] is introduced to replace $F(\phi)$ as follows

$$
\hat{F}(\phi)=\frac{1}{4 \varepsilon} \begin{cases}4(\phi+1)^{2}, & \phi<-1, \\ \left(\phi^{2}-1\right)^{2}, & -1 \leq \phi \leq 1, \\ 4(\phi-1)^{2}, & \phi>1,\end{cases}
$$

We can see that the truncated potential has a quadratic growth for $|\phi|>1$ [32]. Then, we define

$$
\hat{f}(\phi)=\hat{F}^{\prime}(\phi), \quad L_{1}=\max \left|\hat{f}^{\prime}(\phi)\right|=\frac{2}{\varepsilon}, \quad L_{2}=\max \left|g^{\prime \prime}(\phi)\right|=\frac{\sqrt{2} \pi^{2}}{12}\left|\cos \theta_{s}\right| .
$$

A semi-implicit temporal discretization [22] is employed to solve the nonlinear coupled Cahn-Hilliard and Navier-Stokes equations. We define a cut-off function as

$$
\hat{\phi}^{n+1}= \begin{cases}\phi^{n+1} & \left|\phi^{n+1}\right| \leq 1, \\ \operatorname{sign}\left(\phi^{n+1}\right) & \left|\phi^{n+1}\right|>1 .\end{cases}
$$

Given $\phi^{n}, \mathbf{u}^{n}, p^{n}, \rho^{n}$, and $\eta^{n}$, the scheme calculates $\phi^{n+1}, w^{n+1}, \mathbf{u}^{n+1}, p^{n+1}, \rho^{n+1}$ and $\eta^{n+1}$ in two steps. In the first step, we solve the following coupled system to update $\phi^{n+1}, w^{n+1}, \mathbf{u}^{n+1}, \rho^{n+1}$ and $\eta^{n+1}$ :

$$
\left\{\begin{array}{l}
\frac{\phi^{n+1}-\phi^{n}}{\delta t}+\nabla \cdot\left(\mathbf{u}^{n+1} \phi^{n}\right)-M \Delta w^{n+1}=0, \\
w^{n+1}=\lambda\left(-\varepsilon \Delta \phi^{n+1}+\hat{f}\left(\phi^{n}\right)+S_{1}\left(\phi^{n+1}-\phi^{n}\right)\right),
\end{array}\right.
$$




$$
\begin{gathered}
\rho^{n} \frac{\mathbf{u}^{n+1}-\mathbf{u}^{n}}{\delta t}-\nabla \cdot\left(\eta^{n} \nabla \mathbf{u}^{n+1}\right)+\nabla\left(2 p^{n}-p^{n-1}\right)+\rho^{n}\left(\mathbf{u}^{n} \cdot \nabla\right) \mathbf{u}^{n+1}+\mathbf{J}^{n} \cdot \nabla \mathbf{u}^{n+1}+ \\
\phi^{n} \nabla w^{n+1}+\frac{1}{2} \frac{\rho^{n+1}-\rho^{n}}{\delta t} \mathbf{u}^{n+1}+\frac{1}{2} \nabla \cdot\left(\rho^{n} \mathbf{u}^{n}\right) \mathbf{u}^{n+1}+\frac{1}{2} \nabla \cdot\left(\mathbf{J}^{n}\right) \mathbf{u}^{n+1}=0 \\
\rho^{n+1}=\frac{\rho_{1}-\rho_{2}}{2} \hat{\phi}^{n+1}+\frac{\rho_{1}+\rho_{2}}{2}, \\
\eta^{n+1}=\frac{\eta_{1}-\eta_{2}}{2} \hat{\phi}^{n+1}+\frac{\eta_{1}+\eta_{2}}{2},
\end{gathered}
$$

with boundary conditions

$$
\begin{gathered}
\left.\partial_{\mathbf{n}} w^{n+1}\right|_{\Gamma}=0, \\
\left.\left(\frac{\phi^{n+1}-\phi^{n}}{\delta t}+\mathbf{u}_{\tau}^{n+1} \cdot \nabla_{\tau} \phi^{n}+\gamma L^{n+1}\right)\right|_{\Gamma}=0, \\
\left.\left(\mathbf{u}^{n+1} \cdot \mathbf{n}\right)\right|_{\Gamma}=0, \\
\left.\left(\eta^{n} \partial_{n} \boldsymbol{u}_{\tau}^{n+1}+\eta^{n} \beta^{n} \boldsymbol{u}_{s}^{n+1}-\lambda \tilde{L}^{n+1} \nabla_{\tau} \phi^{n}\right)\right|_{\Gamma}=0,
\end{gathered}
$$

where

$$
\begin{gathered}
\tilde{L}^{n+1}=\varepsilon \partial_{\boldsymbol{n}} \phi^{n+1}+g^{\prime}\left(\phi^{n}\right)+S_{2}\left(\phi^{n+1}-\phi^{n}\right), \\
\mathbf{J}^{n}=\frac{\left(\rho_{2}-\rho_{1}\right)}{2} M \nabla w^{n} .
\end{gathered}
$$

In the second step, we update $p^{n+1}$. To avoid the elliptic equation with a variable coefficient $1 / \rho$, the pressure-stabilized scheme $[13,33]$ is adapted to solve the pressure Poisson equation

$$
\left\{\begin{array}{l}
-\Delta\left(p^{n+1}-p^{n}\right)=-\frac{\chi}{\delta t} \nabla \cdot \mathbf{u}^{n+1}, \\
\left.\partial_{\mathbf{n}} p^{n+1}\right|_{\partial \Gamma}=0
\end{array}\right.
$$

where $\chi=\frac{1}{2} \min \left(\rho_{1}, \rho_{2}\right)$.

\subsection{Spatial discretization and energy stability analysis}

A finite difference method on the staggered grids is implemented for the spatial discretization. For a 2-D case, the computational domain is $\Omega=\left[0, L_{x}\right] \times\left[0, L_{y}\right]$, and cell centers $[15,34]$ are located on

$$
x_{i}=\left(i-\frac{1}{2}\right) h_{x}, \quad i=1, \cdots, n_{x}, \quad y_{j}=\left(j-\frac{1}{2}\right) h_{y}, \quad i=1, \cdots, n_{y},
$$

where $h_{x}$ and $h_{y}$ are grid spacings in $x$ and $y$ directions, and $n_{x}$ and $n_{y}$ are the number of grids along the $x$ and $y$ coordinates, respectively. The following finite difference space is introduced to discretize the coupled Cahn-Hilliard and Navier-Stokes system. Ghost cells are also included in these spaces: 


$$
\begin{aligned}
& U_{h}=\left(x_{i-1 / 2}, y_{j}\right) \mid i=1, \cdots, n_{x}+1 ; j=0, \cdots, n_{y}+1, \\
& V_{h}=\left(x_{i}, y_{j-1 / 2}\right) \mid i=0, \cdots, n_{x}+1 ; j=1, \cdots, n_{y}+1, \\
& P_{h}=\left(x_{i}, y_{j}\right) \mid i=0, \cdots, n_{x}+1 ; j=0, \cdots, n_{y}+1, \\
& N_{h}=\left(x_{i-1 / 2}, y_{j-1 / 2}\right) \mid i=1, \cdots, n_{x}+1 ; j=1, \cdots, n_{y}+1,
\end{aligned}
$$

where $P_{h}$ is the cell-centered space, $U_{h}$ and $V_{h}$ are edge-centered space, $(\phi, w, p, \rho, \eta) \in P_{h}, u \in U_{h}$, and $v \in V_{h}$. Some differential and averaged operators [15,34] are defined to complete the spatial discretization. We denote the edge-to-cell difference operators by

$$
d_{x}: U_{h} \rightarrow P_{h} \& N_{h} \rightarrow V_{h} \text { and } d_{y}: V_{h} \rightarrow P_{h} \& N_{h} \rightarrow U_{h},
$$

the center-to-edge difference operators by

$$
D_{x}: P_{h} \rightarrow U_{h} \& V_{h} \rightarrow N_{h} \text { and } D_{y}: P_{h} \rightarrow V_{h} \& U_{h} \rightarrow N_{h},
$$

and the center-to-edge averaged operators by

$$
A_{x}: P_{h} \rightarrow U_{h}, U_{h} \rightarrow P_{h} \& V_{h} \rightarrow N_{h} \text { and } A_{y}: P_{h} \rightarrow V_{h}, V_{h} \rightarrow P_{h} \& U_{h} \rightarrow N_{h} .
$$

With these operators, we can fully discretize the above governing equations. Only the 2-D scheme is presented here, and the 3-D case is similar.

$$
\begin{aligned}
& \phi^{n+1}- \phi^{n}+\delta t\left(d_{x}\left(u^{n+1} A_{x} \phi^{n}\right)+d_{y}\left(v^{n+1} A_{y} \phi^{n}\right)\right)-\delta t M\left(d_{x}\left(D_{x} w^{n+1}\right)+d_{y}\left(D_{y} w^{n+1}\right)\right)=0, \\
& w^{n+1}=-\lambda \varepsilon\left(d_{x}\left(D_{x} \phi^{n+1}\right)+d_{y}\left(D_{y} \phi^{n+1}\right)\right)+\lambda \hat{f}\left(\phi^{n}\right)+\lambda S_{1}\left(\phi^{n+1}-\phi^{n}\right), \\
& A_{x} \rho^{n}\left(u^{n+1}-u^{n}\right)-\delta t\left(D_{x}\left(\eta d_{x} u^{n+1}\right)+d_{y}\left(A_{y}\left(A_{x} \eta\right) D_{y} u^{n+1}\right)\right)+\delta t D_{x}\left(2 p^{n}-p^{n-1}\right) \\
&+\delta t A_{x} \rho^{n}\left(u^{n} D_{x}\left(A_{x} u^{n+1}\right)+A_{x}\left(A_{y} v^{n}\right) d_{y}\left(A_{y} u^{n+1}\right)\right)+\delta t A_{x} \phi^{n} D_{x} w^{n+1} \\
&+\delta t\left(J_{x}^{n} D_{x}\left(A_{x} u^{n+1}\right)+A_{x}\left(A_{y} J_{y}^{n}\right) d_{y}\left(A_{y} u^{n+1}\right)\right)+\frac{1}{2} u^{n+1}\left(A_{x} \rho^{n+1}-A_{x} \rho^{n}\right) \\
&+\frac{1}{2} \delta t\left(D_{x}\left(A_{x} J_{x}^{n}\right)+d_{y}\left(A_{x} J_{y}^{n}\right)\right)+\frac{1}{2} \delta t A_{x} \rho^{n}\left(D_{x}\left(A_{x} u^{n}\right)+d_{y}\left(A_{x} v^{n}\right)\right) u^{n+1}=0, \\
& A_{y} \rho^{n}\left(v^{n+1}-v^{n}\right)-\delta t\left(d_{x}\left(A_{x}\left(A_{y} \eta^{n}\right) D_{x} v^{n+1}\right)+D_{y}\left(\eta^{n} d_{y} v^{n+1}\right)\right)+\delta t D_{y}\left(2 p^{n}-p^{n-1}\right) \\
&+\delta t A_{y} \rho^{n}\left(A_{x}\left(A_{y} u^{n}\right) d_{x}\left(A_{x} v^{n+1}\right)+v^{n} D_{y}\left(A_{y} v^{n+1}\right)\right)+\delta t A_{y} \phi^{n} D_{y} w^{n+1} \\
&+\delta t\left(A_{y}\left(A_{x} J_{x}^{n}\right) d_{x}\left(A_{x} v^{n+1}\right)+J_{y}^{n} D_{y}\left(A_{y} v^{n+1}\right)\right)+\frac{1}{2} v^{n+1}\left(A_{y} \rho^{n+1}-A_{y} \rho^{n}\right) \\
&+\frac{1}{2} \delta t\left(d_{x}\left(A_{y} J_{x}^{n}\right)+D_{y}\left(A_{y} J_{y}^{n}\right)\right)+\frac{1}{2} \delta t A_{y} \rho^{n}\left(d_{x}\left(A_{y} u^{n}\right)+D_{y}\left(A_{y} v^{n}\right)\right) v^{n+1}=0, \\
& \quad d_{x}\left(D_{x}\left(p^{n+1}-p^{n}\right)\right)+d_{y}\left(D_{y}\left(p^{n+1}-p^{n}\right)\right)=\frac{\chi}{\delta t}\left(d_{x} u^{n+1}+d_{y} v^{n+1}\right) .
\end{aligned}
$$

For a rectangular domain, the east and west boundaries can be denoted by $\Gamma_{\mathrm{EW}}$, and the north and south boundaries can be denoted by $\Gamma_{\mathrm{NS}}$. An operator $\mathbf{D}=\left(D_{x}, D_{y}\right)$ is introduced to simplify the discretization. The fully discrete boundary conditions are given based on these operators: 


$$
\left\{\begin{array}{l}
\left.\left(\mathbf{D} w^{n+1} \cdot \mathbf{n}\right)\right|_{\Gamma}=0, \\
\left.\left(\phi^{n+1}-\phi^{n}\right)\right|_{\Gamma_{N S}}+\left.\delta t A_{x}\left(A_{y} u^{n+1}\right) d_{x}\left(A_{x}\left(A_{y} \phi^{n}\right)\right)\right|_{\Gamma_{N S}}+\left.\gamma \tilde{L}^{n+1}\right|_{\Gamma_{N S}}=0, \\
\left.\left(\phi^{n+1}-\phi^{n}\right)\right|_{\Gamma_{E W}}+\left.\delta t A_{y}\left(A_{x} v^{n+1}\right) d_{y}\left(A_{y}\left(A_{x} \phi^{n}\right)\right)\right|_{\Gamma_{E W}}+\left.\gamma \tilde{L}^{n+1}\right|_{\Gamma_{E W}}=0, \\
\left.\left(\mathbf{u}^{n+1} \cdot \mathbf{n}\right)\right|_{\Gamma}=0, \\
\left.\left(\sigma_{N S} A_{y}\left(A_{x} \eta^{n}\right) D_{y} u^{n+1}+A_{y}\left(A_{x}\left(\eta^{n} \beta^{n}\right) u^{n+1}\right)-\lambda A_{x} \tilde{L}^{n+1} D_{x}\left(A_{y} \phi^{n}\right)\right)\right|_{\Gamma_{N S}}=0, \\
\left.\left(\sigma_{E W} A_{x}\left(A_{y} \eta^{n}\right) D_{x} v^{n+1}+A_{x}\left(A_{y}\left(\eta^{n} \beta^{n}\right) v^{n+1}\right)-\lambda A_{y} \tilde{L}^{n+1} D_{y}\left(A_{x} \phi^{n}\right)\right)\right|_{\Gamma_{E W}}=0, \\
\left.\left(\mathbf{D} p^{n+1} \cdot \mathbf{n}\right)\right|_{\Gamma}=0
\end{array}\right.
$$

where

$$
\begin{aligned}
& \left\{\begin{array}{l}
\left.\left(\tilde{L}^{n+1}-g^{\prime}\left(\phi^{n}\right)-S_{2}\left(\phi^{n+1}-\phi^{n}\right)\right)\right|_{\Gamma_{N S}}-\left.\sigma_{N S} \varepsilon D_{y} \phi^{n+1}\right|_{\Gamma_{N S}}=0, \\
\left.\left(\tilde{L}^{n+1}-g^{\prime}\left(\phi^{n}\right)-S_{2}\left(\phi^{n+1}-\phi^{n}\right)\right)\right|_{\Gamma_{E W}}-\left.\sigma_{E W} \varepsilon D_{x} \phi^{n+1}\right|_{\Gamma_{E W}}=0,
\end{array}\right. \\
& \rho^{n+1}=\frac{\rho_{1}-\rho_{2}}{2} \hat{\phi}^{n+1}+\frac{\rho_{1}+\rho_{2}}{2}, \quad \eta^{n+1}=\frac{\eta_{1}-\eta_{2}}{2} \hat{\phi}^{n+1}+\frac{\eta_{1}+\eta_{2}}{2}, \\
& J_{x}^{n}=\frac{\left(\rho_{2}-\rho_{1}\right)}{2} M D_{x} w^{n}, \quad J_{y}^{n}=\frac{\left(\rho_{2}-\rho_{1}\right)}{2} M D_{y} w^{n}, \\
& \sigma_{N S}=\left\{\begin{array}{lc}
1, & \text { on } \Gamma_{N} \\
-1, & \text { on } \Gamma_{S}
\end{array}, \quad \sigma_{E W}=\left\{\begin{array}{ll}
1, & \text { on } \Gamma_{E} \\
-1, & \text { on } \Gamma_{W}
\end{array},\right.\right.
\end{aligned}
$$

where $J_{x} \in U_{h}, J_{y} \in V_{h}$.

In the component form, the cell-centered functions (e.g., $\phi$ and $\varphi \in P_{h}$ ) can be identified via $\phi_{i, j}=\phi\left(x_{i}, y_{j}\right)$. The edge-centered functions in the $U_{h}$ space (e.g., $f$ and $\left.g \in U_{h}\right)$ can be identified via $f_{i+1 / 2, j}=f\left(x_{i+1 / 2}, y_{j}\right)$. The edge-centered functions in the $V_{h}$ space (e.g., $f$ and $\left.g \in V_{h}\right)$ can be identified via $g_{i+1 / 2, j}=g\left(x_{i+1 / 2}, y_{j}\right)$. To simplify the proof of energy decay property of the numerical scheme, the following weighted inner products (cf. [15, 34]) are defined as follows:

$$
\begin{gathered}
\langle\phi, \varphi\rangle_{P_{h}}=\sum_{i=1}^{n x} \sum_{j=1}^{n y} \phi_{i, j} \varphi_{i, j}, \quad \phi, \varphi \in P_{h}, \\
\langle f, g\rangle_{U_{h}}=\frac{1}{2} \sum_{i=1}^{n x} \sum_{j=1}^{n y}\left(f_{i+1 / 2, j} g_{i+1 / 2, j}+f_{i-1 / 2, j} g_{i-1 / 2, j}\right), \quad f, g \in U_{h}, \\
\langle f, g\rangle_{V_{h}}=\frac{1}{2} \sum_{i=1}^{n x} \sum_{j=1}^{n y}\left(f_{i, j+1 / 2} g_{i, j+1 / 2}+f_{i, j-1 / 2} g_{i, j-1 / 2}\right), \quad f, g \in V_{h} .
\end{gathered}
$$

The following norms are defined for the cell-centered functions which belong to the space $P_{h}$,

$$
\|\phi\|_{2}=\left(h_{x} h_{y} \sum_{i=1}^{n x} \sum_{j=1}^{n y}\left|\phi_{i, j}\right|^{2}\right)^{1 / 2} \text {. }
$$

For any $\phi \in P_{h}$, we define

$$
\|\nabla \phi\|_{2}=\sqrt{h_{x} h_{y}\left\langle D_{x} \phi, D_{x} \phi\right\rangle_{U_{h}}+h_{x} h_{y}\left\langle D_{y} \phi, D_{y} \phi\right\rangle_{V_{h}}} .
$$

For the edge-centered cell velocity $\mathbf{u}$, we define

$$
\|\mathbf{u}\|_{2}=\sqrt{h_{x} h_{y}\langle u, u\rangle_{U_{h}}+h_{x} h_{y}\langle v, v\rangle_{V_{h}}},
$$


where $u \in U_{h}$, and $v \in V_{h}$. The following summation-by-parts formulae [15, 27, 34] are important to prove the energy decay property of the fully discrete scheme.

Lemma 3.1. If $\phi \in P_{h}$ and $f \in U_{h}$, then

$$
\left\{\begin{array}{l}
h_{x} h_{y}\left\langle D_{x} \phi, f\right\rangle_{U_{h}}=-h_{x} h_{y}\left\langle\phi, d_{x} f\right\rangle_{P_{h}}+h_{x} h_{y}\langle f, \phi\rangle_{\Gamma_{E W}}, \\
\langle f, \phi\rangle_{\Gamma_{E W}}=-\left(1 / h_{x}\right) \sum_{j=1}^{n y} A_{x} \phi_{1 / 2, j} f_{1 / 2, j}+\left(1 / h_{x}\right) \sum_{j=1}^{n y} A_{x} \phi_{n x+1 / 2, j} f_{n x+1 / 2, j} .
\end{array}\right.
$$

Proof. Clearly, by the definition of $D_{x}$, we have

$$
\begin{aligned}
& h_{x} h_{y}\left\langle D_{x} \phi, f\right\rangle_{U_{h}}=\frac{h_{x} h_{y}}{2} \sum_{i=1}^{n x} \sum_{j=1}^{n y}\left(D_{x} \phi_{i+1 / 2, j} f_{i+1 / 2, j}+D_{x} \phi_{i-1 / 2, j} f_{i-1 / 2, j}\right) \\
= & \frac{h_{y}}{2} \sum_{i=1}^{n x} \sum_{j=1}^{n y}\left(\left(\phi_{i+1, j}-\phi_{i, j}\right) f_{i+1 / 2, j}+\left(\phi_{i, j}-\phi_{i-1, j}\right) f_{i-1 / 2, j}\right) \\
= & \frac{h_{y}}{2} \sum_{i=1}^{n x} \sum_{j=1}^{n y}\left(\left(\phi_{i+1, j}-\phi_{i, j}\right) f_{i+1 / 2, j}+\left(\phi_{i, j}-\phi_{i-1, j}\right) f_{i-1 / 2, j}\right) \\
= & \frac{h_{y}}{2} \sum_{i=1}^{n x} \sum_{j=1}^{n y}\left(\phi_{i, j} f_{i-1 / 2, j}-\phi_{i, j} f_{i+1 / 2, j}+\phi_{i, j} f_{i-1 / 2, j}-\phi_{i, j} f_{i+1 / 2, j}\right) \\
& -\frac{h_{y}}{2} \sum_{j=1}^{n y} \phi_{0, j} f_{1 / 2, j}-\frac{h_{y}}{2} \sum_{j=1}^{n y} \phi_{1, j} f_{1 / 2, j}+\frac{h_{y}}{2} \sum_{j=1}^{n y} \phi_{n x, j} f_{n x+1 / 2, j}+\frac{h_{y}}{2} \sum_{j=1}^{n y} \phi_{n x+1, j} f_{n x+1 / 2, j},
\end{aligned}
$$

where $\phi_{0, j}$ and $\phi_{n x+1, j}$ are the values of ghost cells. Then by the definition of $A_{x}$, we can easily derive

$$
\begin{aligned}
& h_{x} h_{y}\left\langle D_{x} \phi, f\right\rangle_{U_{h}} \\
= & -h_{x} h_{y} \sum_{i=1}^{n x} \sum_{j=1}^{n y}\left(\phi_{i, j} \frac{f_{i+1 / 2, j}-f_{i-1 / 2, j}}{h_{x}}\right)-h_{y} \sum_{j=1}^{n y} A_{x} \phi_{1 / 2, j} f_{1 / 2, j}+h_{y} \sum_{j=1}^{n y} A_{x} \phi_{n x+1 / 2, j} f_{n x+1 / 2, j} \\
= & -h_{x} h_{y}\left\langle\phi, d_{x} f\right\rangle_{P_{h}}-h_{y} \sum_{j=1}^{n y} A_{x} \phi_{1 / 2, j} f_{1 / 2, j}+h_{y} \sum_{j=1}^{n y} A_{x} \phi_{n x+1 / 2, j} f_{n x+1 / 2, j} .
\end{aligned}
$$

Now we can conclude the desired result.

Similarly, we can derive the other summation-by-parts formulae. If $\phi \in P_{h}$ and $f \in V_{h}$, then

$$
\left\{\begin{array}{l}
h_{x} h_{y}\left\langle D_{y} \phi, f\right\rangle_{V_{h}}=-h_{x} h_{y}\left\langle\phi, d_{y} f\right\rangle_{P_{h}}+h_{x} h_{y}\langle f, \phi\rangle_{\Gamma_{N s}}, \\
\langle f, \phi\rangle_{\Gamma_{N S}}=-\left(1 / h_{y}\right) \sum_{i=1}^{n x} A_{y} \phi_{i, 1 / 2} f_{i, 1 / 2}+\left(1 / h_{y}\right) \sum_{i=1}^{n x} A_{y} \phi_{i, n y+1 / 2} f_{i, n y+1 / 2}
\end{array}\right.
$$

If $f \in N_{h}$, and $g \in U_{h}$, then

$$
\left\{\begin{array}{l}
h_{x} h_{y}\left\langle d_{y} f, g\right\rangle_{U_{h}}=-h_{x} h_{y}\left\langle f, D_{y} g\right\rangle_{N_{h}}+h_{x} h_{y}\langle f, g\rangle_{\Gamma_{N S}}, \\
\langle f, g\rangle_{\Gamma_{N S}}=-\left(1 / h_{y}\right) \sum_{i=1}^{n x+1} A_{y} g_{i-1 / 2,1 / 2} f_{i-1 / 2,1 / 2}+\left(1 / h_{y}\right) \sum_{i=1}^{n x+1} A_{y} g_{i-1 / 2, n y+1 / 2} f_{i-1 / 2, n y+1 / 2} .
\end{array}\right.
$$

If $f \in N_{h}$ and $g \in V_{h}$, then

$$
\left\{\begin{array}{l}
h_{x} h_{y}\left\langle d_{x} f, g\right\rangle_{V_{h}}=-h_{x} h_{y}\left\langle f, D_{x} g\right\rangle_{N_{h}}+h_{x} h_{y}\langle f, g\rangle_{\Gamma_{E W}}, \\
\langle f, g\rangle_{\Gamma_{E W}}=-\left(1 / h_{x}\right) \sum_{j=1}^{n y+1} A_{x} g_{1 / 2, j-1 / 2} f_{1 / 2, j-1 / 2}+\left(1 / h_{x}\right) \sum_{j=1}^{n y+1} A_{x} g_{n x+1 / 2, j-1 / 2} f_{n x+1 / 2, j-1 / 2} .
\end{array}\right.
$$


To simplify the proof of energy decay property, the following two definitions are also introduced to deal with boundary conditions. If $f \in U_{h}$, and $g \in U_{h}$, then

$$
\langle f, g\rangle_{\Gamma_{E W}}=-\left(1 / h_{x}\right) \sum_{j=1}^{n y} g_{1 / 2, j} f_{1 / 2, j}+\left(1 / h_{x}\right) \sum_{j=1}^{n v} g_{n x+1 / 2, j} f_{n x+1 / 2, j} .
$$

If $f \in V_{h}$, and $g \in V_{h}$, then

$$
\langle f, g\rangle_{\Gamma_{N S}}=-\left(1 / h_{y}\right) \sum_{i=1}^{n x} g_{i, 1 / 2} f_{i, 1 / 2}+\left(1 / h_{y}\right) \sum_{i=1}^{n x} g_{i, n y+1 / 2} f_{i, n y+1 / 2} .
$$

Lemma 3.2. For any variables $\left(u^{n}, \tilde{u}^{n+1}\right) \in U_{h}$ and $\left(v^{n}, \tilde{v}^{n+1}\right) \in V_{h}$, let $\left(u^{n}, v^{n}\right)$ be denoted by $\mathbf{u}^{\mathrm{n}}$. If $\mathbf{u}^{n} \cdot \mathbf{n}=0$ on boundaries, we have

$$
\begin{aligned}
& \left\langle u^{n} D_{x}\left(A_{x} \tilde{u}^{n+1}\right)+A_{x}\left(A_{y} v^{n}\right) d_{y}\left(A_{y} \tilde{u}^{n+1}\right), \tilde{u}^{n+1}\right\rangle_{U_{h}}+\left\langle A_{x}\left(A_{y} u^{n}\right) d_{x}\left(A_{x} \tilde{v}^{n+1}\right)+v^{n} D_{y}\left(A_{y} \tilde{v}^{n+1}\right), \tilde{v}^{n+1}\right\rangle_{V_{h}} \\
& +\frac{1}{2}\left\langle\left(D_{x}\left(A_{x} u^{n}\right)+d_{y}\left(A_{x} v^{n}\right)\right) \tilde{u}^{n+1}, \tilde{u}^{n+1}\right\rangle_{U_{h}}+\frac{1}{2}\left\langle\left(d_{x}\left(A_{y} u^{n}\right)+D_{y}\left(A_{y} v^{n}\right)\right) \tilde{v}^{n+1}, \tilde{v}^{n+1}\right\rangle_{V_{h}}=0 .
\end{aligned}
$$

Proof. Using the inner product formula (3.24), we have

$$
\begin{aligned}
& \left\langle u^{n} D_{x}\left(A_{x} \tilde{u}^{n+1}\right), \tilde{u}^{n+1}\right\rangle_{U_{h}}+\frac{1}{2}\left\langle D_{x}\left(A_{x} u^{n}\right) \tilde{u}^{n+1}, \tilde{u}^{n+1}\right\rangle_{U_{h}} \\
= & \frac{1}{2} \sum_{i=1}^{n x} \sum_{j=1}^{n y}\left(u_{i+1 / 2, j}^{n} D_{x}\left(A_{x} \tilde{u}_{i+1 / 2, j}^{n+1}\right) \tilde{u}_{i+1 / 2, j}^{n+1}+u_{i-1 / 2, j}^{n} D_{x}\left(A_{x} \tilde{u}_{i-1 / 2, j}^{n+1}\right) \tilde{u}_{i-1 / 2, j}^{n+1}\right) \\
& +\frac{1}{4} \sum_{i=1}^{n x} \sum_{j=1}^{n y}\left(D_{x}\left(A_{x} u_{i+1 / 2, j}^{n+1}\right)\left(\tilde{u}_{i+1 / 2, j}^{n+1}\right)^{2}+D_{x}\left(A_{x} u_{i-1 / 2, j}^{n}\right)\left(\tilde{u}_{i-1 / 2, j}^{n+1}\right)^{2}\right) \\
= & \frac{1}{8} \sum_{i=1}^{n x} \sum_{j=1}^{n y}\left(u_{i+1 / 2, j}^{n} \frac{\tilde{u}_{i+3 / 2, j}^{n+1}-\tilde{u}_{i-1 / 2, j}^{n+1}}{h_{x}}\left(\tilde{u}_{i+3 / 2, j}^{n+1}+\tilde{u}_{i-1 / 2, j}^{n+1}\right)\right. \\
& +u_{i-1 / 2, j}^{n} \frac{\left.\tilde{u}_{i+1 / 2, j}^{n+1}-\tilde{u}_{i-3 / 2, j}^{n+1}\left(\tilde{u}_{i+1 / 2, j}^{n+1}+\tilde{u}_{i-3 / 2, j}^{n+1}\right)\right)}{h_{x}} \\
& +\frac{1}{8} \sum_{i=1}^{n x} \sum_{j=1}^{n y}\left(\frac{\left(u_{i+3 / 2, j}^{n}+u_{i+1 / 2, j}^{n}\right)-\left(u_{i+1 / 2, j}^{n}+u_{i-1 / 2, j}^{n}\right)}{h_{x}}\left(\tilde{u}_{i+1 / 2, j}^{n+1}\right)^{2}\right. \\
& \left.+\frac{\left(u_{i+1 / 2, j}^{n}+u_{i-1 / 2, j}^{n}\right)-\left(u_{i-1 / 2, j}^{n}+u_{i-3 / 2, j}^{n}\right)}{h_{x}}\left(\tilde{u}_{i-1 / 2, j}^{n+1}\right)^{2}\right) \\
= & \frac{1}{8 h_{x}} \sum_{i=1}^{n x} \sum_{j=1}^{n y}\left(u_{i+1 / 2, j}^{n}\left(\left(\tilde{u}_{i+3 / 2, j}^{n+1}\right)^{2}-\left(\tilde{u}_{i-1 / 2, j}^{n+1}\right)^{2}\right)+u_{i-1 / 2, j}^{n}\left(\left(\tilde{u}_{i+1 / 2, j}^{n+1}\right)^{2}-\left(\tilde{u}_{i-3 / 2, j}^{n+1}\right)^{2}\right)\right) \\
& +\frac{1}{8 h_{x}} \sum_{i=1}^{n x} \sum_{j=1}^{n y}\left(u_{i+1 / 2}^{n}\left(\left(\tilde{u}_{i-1 / 2, j}^{n+1}\right)^{2}-\left(\tilde{u}_{i+3 / 2, j}^{n+1}\right)^{2}\right)+u_{i-1 / 2}^{n}\left(\left(\tilde{u}_{i-3 / 2, j}^{n+1}\right)^{2}-\left(\tilde{u}_{i+1 / 2, j}^{n+1}\right)^{2}\right)\right)=0 .
\end{aligned}
$$

Similarly, we can derive

$$
\left\{\begin{array}{l}
\left\langle A_{x}\left(A_{y} v^{n}\right) d_{y}\left(A_{y} \tilde{u}^{n+1}\right), \tilde{u}^{n+1}\right\rangle_{U_{h}}+\frac{1}{2}\left\langle d_{y}\left(A_{x} v^{n}\right) \tilde{u}^{n+1}, \tilde{u}^{n+1}\right\rangle_{U_{h}}=0, \\
\left\langle A_{x}\left(A_{y} u^{n}\right) d_{x}\left(A_{x} \tilde{v}^{n+1}\right), \tilde{v}^{n+1}\right\rangle_{V_{h}}+\frac{1}{2}\left\langle d_{x}\left(A_{y} u^{n}\right) \tilde{v}^{n+1}, \tilde{v}^{n+1}\right\rangle_{V_{h}}=0, \\
\left\langle v^{n} D_{y}\left(A_{y} \tilde{v}^{n+1}\right), \tilde{v}^{n+1}\right\rangle_{V_{h}}+\frac{1}{2}\left\langle D_{y}\left(A_{y} v^{n}\right) \tilde{v}^{n+1}, \tilde{v}^{n+1}\right\rangle_{V_{h}}=0 .
\end{array}\right.
$$

Now the desired result can be easily obtained based on the above four equalities.

We then introduce some energy terms as follows: 


$$
E_{k c}^{n}=\frac{1}{2}\left\|\sigma^{n} \boldsymbol{u}^{n}\right\|_{2}^{2}, \quad E_{s}^{n}=\lambda\left(\frac{\varepsilon}{2}\left\|\nabla \phi^{n}\right\|_{2}^{2}+\left(\hat{F}\left(\phi^{n}\right), 1\right)\right), \quad E_{w f}^{n}=\lambda\left(g\left(\phi^{n}\right), 1\right)_{\Gamma},
$$

where

$$
\begin{gathered}
\left(\hat{F}\left(\phi^{n}\right), 1\right)=h_{x} h_{y}\left\langle\hat{F}\left(\phi^{n}\right), 1\right\rangle_{P_{h}}, \\
\left(g\left(\phi^{n}\right), 1\right)_{\Gamma}=h_{x} h_{y}\left\langle\sigma_{N S} g\left(\phi^{n}\right), 1\right\rangle_{\Gamma_{N S}}+h_{x} h_{y}\left\langle\sigma_{E W} g\left(\phi^{n}\right), 1\right\rangle_{\Gamma_{E W}},
\end{gathered}
$$

$E_{k c}^{n}$ is the bulk kinetic energy, $E_{s}^{n}$ is the bulk free energy, and $E_{w f}^{n}$ is the surface energy at the fluid-solid boundary.

Theorem 3.1. Assume $\mathbf{u}_{\mathbf{w}}=0, S_{1} \geq L_{1} / 2$ and $S_{2} \geq L_{2} / 2$, then the fully discrete scheme (3.13) - (3.18) is energy stable and satisfies the following discrete energy law

$$
E^{n+1}-E^{n} \leq-\delta t\left\|\sqrt{\eta^{n}} \nabla \mathbf{u}^{n+1}\right\|_{2}^{2}-\delta t M\left\|\nabla w^{n+1}\right\|_{2}^{2}-\delta t\left\|\sqrt{\eta^{n} \beta^{n}} \mathbf{u}_{\mathrm{s}}^{n+1}\right\|_{\Gamma}^{2}-\lambda \delta t \gamma\left\|\tilde{L}^{n+1}\right\|_{\Gamma}^{2}
$$

where

$$
E^{n}=E_{k c}^{n}+E_{s}^{n}+E_{w f}^{n}+\frac{\delta t^{2}}{2 \chi}\left\|\nabla p^{n}\right\|_{2}^{2}
$$

here $E^{n}$ is the total energy of the whole system at the $n^{\text {th }}$ time step.

Proof. Using the Lemma 3.2, we have

$$
\begin{aligned}
\delta t & \left\langle A_{x} \rho^{n}\left(u^{n} D_{x}\left(A_{x} u^{n+1}\right)+A_{x}\left(A_{y} v^{n}\right) d_{y}\left(A_{y} u^{n+1}\right)\right), u^{n+1}\right\rangle_{U_{h}} \\
& +\delta t\left\langle A_{y} \rho^{n}\left(A_{x}\left(A_{y} u^{n}\right) d_{x}\left(A_{x} v^{n+1}\right)+v^{n} D_{y}\left(A_{y} v^{n+1}\right)\right), v^{n+1}\right\rangle_{V_{h}} \\
& +\frac{1}{2} \delta t\left\langle A_{x} \rho^{n}\left(D_{x}\left(\rho^{n} A_{x} u^{n}\right)+d_{y}\left(A_{x} v^{n}\right)\right) u^{n+1}, u^{n+1}\right\rangle_{U_{h}} \\
& +\frac{1}{2} \delta t\left\langle A_{y} \rho^{n}\left(d_{x}\left(A_{y} u^{n}\right)+D_{y}\left(A_{y} v^{n}\right)\right) v^{n+1}, v^{n+1}\right\rangle_{V_{h}}=0,
\end{aligned}
$$

and

$$
\begin{aligned}
\delta & t\left\langle\left(J_{x}^{n} D_{x}\left(A_{x} u^{n+1}\right)+A_{x}\left(A_{y} J_{y}^{n}\right) d_{y}\left(A_{y} u^{n+1}\right)\right), u^{n+1}\right\rangle_{U_{h}} \\
& +\delta t\left\langle\left(A_{y}\left(A_{x} J_{x}^{n}\right) d_{x}\left(A_{x} v^{n+1}\right)+J_{y}^{n} D_{y}\left(A_{y} v^{n+1}\right)\right), v^{n+1}\right\rangle_{V_{h}} \\
& +\frac{1}{2} \delta t\left\langle\left(D_{x}\left(A_{x} J_{x}^{n}\right)+d_{y}\left(A_{x} J_{y}^{n}\right)\right), u^{n+1}\right\rangle_{U_{h}}+\frac{1}{2} \delta t\left\langle\left(d_{x}\left(A_{y} J_{x}^{n}\right)+D_{y}\left(A_{y} J_{y}^{n}\right)\right), v^{n+1}\right\rangle_{V_{h}}=0 .
\end{aligned}
$$

(i) Taking the discrete inner-product of (3.15) and (3.16) with $2 u^{n+1}$ and $2 v^{n+1}$ respectively, 


$$
\begin{aligned}
& \left\langle\sigma_{x}^{n} u^{n+1}, \sigma_{x}^{n} u^{n+1}\right\rangle_{U_{h}}-\left\langle\sigma_{x}^{n} u^{n}, \sigma_{x}^{n} u^{n}\right\rangle_{U_{h}}+\left\langle\sigma_{x}^{n}\left(u^{n+1}-u^{n}\right), \sigma_{x}^{n}\left(u^{n+1}-u^{n}\right)\right\rangle_{U_{h}} \\
& +\left\langle\sigma_{y}^{n} v^{n+1}, \sigma_{y}^{n} v^{n+1}\right\rangle_{V_{h}}-\left\langle\sigma_{y}^{n} v^{n}, \sigma_{y}^{n} v^{n}\right\rangle_{V_{h}}+\left\langle\sigma_{y}^{n}\left(v^{n+1}-v^{n}\right), \sigma_{y}^{n}\left(v^{n+1}-v^{n}\right)\right\rangle_{V_{h}} \\
& +\left\langle\sigma_{x}^{n+1} u^{n+1}, \sigma_{x}^{n+1} u^{n+1}\right\rangle_{U_{h}}-\left\langle\sigma_{x}^{n} u^{n+1}, \sigma_{x}^{n} u^{n+1}\right\rangle_{U_{h}}+\left\langle\sigma_{y}^{n+1} v^{n+1}, \sigma_{y}^{n+1} v^{n+1}\right\rangle_{V_{h}} \\
& -\left\langle\sigma_{y}^{n} v^{n+1}, \sigma_{y}^{n} v^{n+1}\right\rangle_{V_{h}}+2 \delta t\left\langle p^{n+1}-2 p^{n}+p^{n-1}, d_{x} u^{n+1}\right\rangle_{P_{h}}-2 \delta t\left\langle p^{n+1}, d_{x} u^{n+1}\right\rangle_{P_{n}} \\
& +2 \delta t\left\langle p^{n+1}-2 p^{n}+p^{n-1}, d_{y} v^{n+1}\right\rangle_{P_{h}}-2 \delta t\left\langle p^{n+1}, d_{y} v^{n+1}\right\rangle_{P_{n}} \\
& +2 \delta t\left\langle\sqrt{\eta^{n}} d_{x} u^{n+1}, \sqrt{\eta^{n}} d_{x} u^{n+1}\right\rangle_{P_{h}}+2 \delta t\left\langle\sqrt{A_{y}\left(A_{x} \eta^{n}\right)} D_{y} u^{n+1}, \sqrt{A_{y}\left(A_{x} \eta^{n}\right)} D_{y} u^{n+1}\right\rangle_{N_{h}} \\
& +2 \delta t\left\langle\sqrt{A_{x}\left(A_{y} \eta^{n}\right)} D_{x} v^{n+1}, \sqrt{A_{x}\left(A_{y} \eta^{n}\right)} D_{x} v^{n+1}\right\rangle_{N_{h}}+2 \delta t\left\langle\sqrt{\eta^{n}} d_{y} v^{n+1}, \sqrt{\eta^{n}} d_{y} v^{n+1}\right\rangle_{P_{n}} \\
& +2 \delta t\left\langle A_{x} \phi^{n} D_{x} w^{n+1}, u^{n+1}\right\rangle_{U_{h}}+2 \delta t\left\langle A_{y} \phi^{n} D_{y} w^{n+1}, v^{n+1}\right\rangle_{V_{n}} \\
& -2 \delta t\left\langle A_{y}\left(A_{x} \eta^{n}\right) D_{y} u^{n+1}, u^{n+1}\right\rangle_{\Gamma_{N S}}-2 \delta t\left\langle A_{x}\left(A_{y} \eta^{n}\right) D_{x} v^{n+1}, v^{n+1}\right\rangle_{\Gamma_{E W}}=0 .
\end{aligned}
$$

(ii) Taking the discrete inner-product of (3.17) with $2 \delta t^{2}\left(p^{\mathrm{n}+1}-2 p^{n}+p^{\mathrm{n}-1}\right) / \chi$, we can derive

$$
\begin{aligned}
& \left\langle d_{x}\left(D_{x}\left(p^{n+1}-p^{n}\right)\right), \frac{2 \delta t^{2}}{\chi}\left(p^{n+1}-2 p^{n}+p^{n-1}\right)\right\rangle_{P_{h}} \\
& +\left\langle d_{y}\left(D_{y}\left(p^{n+1}-p^{n}\right)\right), \frac{2 \delta t^{2}}{\chi}\left(p^{n+1}-2 p^{n}+p^{n-1}\right)\right\rangle_{P_{h}} \\
= & \frac{\delta t^{2}}{\chi}\left\langle D_{x}\left(p^{n+1}-2 p^{n}+p^{n-1}\right), 2 D_{x}\left(p^{n+1}-p^{n}\right)\right\rangle_{U_{h}} \\
& +\frac{\delta t^{2}}{\chi}\left\langle D_{y}\left(p^{n+1}-2 p^{n}+p^{n-1}\right), 2 D_{y}\left(p^{n+1}-p^{n}\right)\right\rangle_{V_{h}} \\
= & -\frac{\delta t^{2}}{\chi}\left(\left\langle D_{x}\left(p^{n+1}-p^{n}\right), D_{x}\left(p^{n+1}-p^{n}\right)\right\rangle_{U_{h}}-<D_{x}\left(p^{n}-p^{n-1}\right), D_{x}\left(p^{n}-p^{n-1}\right)\right\rangle_{U_{h}} \\
& \left.+\left\langle D_{x}\left(p^{n+1}-2 p^{n}+p^{n-1}\right), D_{x}\left(p^{n+1}-2 p^{n}+p^{n-1}\right)\right\rangle_{U_{h}}\right) \\
& -\frac{\delta t^{2}}{\chi}\left(\left\langle D_{y}\left(p^{n+1}-p^{n}\right), D_{y}\left(p^{n+1}-p^{n}\right)\right\rangle_{V_{h}}-\left\langle D_{y}\left(p^{n}-p^{n-1}\right), D_{y}\left(p^{n}-p^{n-1}\right)\right\rangle_{V_{h}}\right. \\
& \left.+\left\langle D_{y}\left(p^{n+1}-2 p^{n}+p^{n-1}\right), D_{y}\left(p^{n+1}-2 p^{n}+p^{n-1}\right)\right\rangle_{V_{n}}\right) \\
= & 2 \delta t\left\langle p^{n+1}-2 p^{n}+p^{n-1}, d_{x} u^{n+1}\right\rangle_{P_{h}}+2 \delta t\left\langle p^{n+1}-2 p^{n}+p^{n-1}, d_{y} v^{n+1}\right\rangle_{P_{n}} .
\end{aligned}
$$

(iii) Taking the discrete inner-product of (3.17) with $-2 \delta t^{2} p^{n+1} / \chi$, we have

$$
\begin{aligned}
& \frac{\delta t^{2}}{\chi}\left(\left\langle D_{x} p^{n+1}, D_{x} p^{n+1}\right\rangle_{U_{h}}-\left\langle D_{x} p^{n}, D_{x} p^{n}\right\rangle_{U_{h}}+\left\langle D_{x}\left(p^{n+1}-p^{n}\right), D_{x}\left(p^{n+1}-p^{n}\right)\right\rangle_{U_{h}}\right) \\
& +\frac{\delta t^{2}}{\chi}\left(\left\langle D_{y} p^{n+1}, D_{y} p^{n+1}\right\rangle_{V_{h}}-\left\langle D_{y} p^{n}, D_{y} p^{n}\right\rangle_{V_{h}}+\left\langle D_{y}\left(p^{n+1}-p^{n}\right), D_{y}\left(p^{n+1}-p^{n}\right)\right\rangle_{V_{h}}\right) \\
= & -2 \delta t\left\langle p^{n+1}, d_{x} u^{n+1}\right\rangle_{P_{h}}-2 \delta t\left\langle p^{n+1}, d_{y} v^{n+1}\right\rangle_{P_{h}} .
\end{aligned}
$$

(iv) Now summing up equations (3.33) and (3.34), we get 


$$
\begin{aligned}
& \frac{\delta t^{2}}{\chi}\left(\left\langle D_{x}\left(p^{n}-p^{n-1}\right), D_{x}\left(p^{n}-p^{n-1}\right)\right\rangle_{U_{h}}+\left\langle D_{y}\left(p^{n}-p^{n-1}\right), D_{y}\left(p^{n}-p^{n-1}\right)\right\rangle_{V_{h}}\right) \\
& +\frac{\delta t^{2}}{\chi}\left(\left\langle D_{x} p^{n+1}, D_{x} p^{n+1}\right\rangle_{U_{h}}-\left\langle D_{x} p^{n}, D_{x} p^{n}\right\rangle_{U_{h}}+\left\langle D_{y} p^{n+1}, D_{y} p^{n+1}\right\rangle_{V_{h}}-\left\langle D_{y} p^{n}, D_{y} p^{n}\right\rangle_{V_{h}}\right) \\
= & \frac{\delta t^{2}}{\chi}\left\langle D_{x}\left(p^{n+1}-2 p^{n}+p^{n-1}\right), D_{x}\left(p^{n+1}-2 p^{n}+p^{n-1}\right)\right\rangle_{U_{h}} \\
& +\frac{\delta t^{2}}{\chi}\left\langle D_{y}\left(p^{n+1}-2 p^{n}+p^{n-1}\right), D_{y}\left(p^{n+1}-2 p^{n}+p^{n-1}\right)\right\rangle_{V_{h}} \\
& +2 \delta t\left\langle p^{n+1}-2 p^{n}+p^{n-1}, d_{x} u^{n+1}\right\rangle_{P_{h}}+2 \delta t\left\langle p^{n+1}-2 p^{n}+p^{n-1}, d_{y} v^{n+1}\right\rangle_{P_{h}} \\
& -2 \delta t\left\langle p^{n+1}, d_{x} u^{n+1}\right\rangle_{P_{h}}-2 \delta t\left\langle p^{n+1}, d_{y} v^{n+1}\right\rangle_{P_{h}} .
\end{aligned}
$$

(v) Next, we take the difference of (3.17) at the time step $t^{n+1}$ and $t^{n}$ to derive

$$
\begin{aligned}
& \frac{\delta t^{2}}{\chi}\left\langle D_{x}\left(p^{n+1}-2 p^{n}+p^{n-1}\right), D_{x}\left(p^{n+1}-2 p^{n}+p^{n-1}\right)\right\rangle_{U_{h}} \\
& +\frac{\delta t^{2}}{\chi}\left\langle D_{y}\left(p^{n+1}-2 p^{n}+p^{n-1}\right), D_{y}\left(p^{n+1}-2 p^{n}+p^{n-1}\right)\right\rangle_{V_{h}} \\
\leq & \chi\left\langle u^{n+1}-u^{n}, u^{n+1}-u^{n}\right\rangle_{U_{h}}+\chi\left\langle v^{n+1}-v^{n}, v^{n+1}-v^{n}\right\rangle_{U_{h}} \\
\leq & \frac{1}{2}\left\langle\sigma_{x}^{n}\left(u^{n+1}-u^{n}\right), \sigma_{x}^{n}\left(u^{n+1}-u^{n}\right)\right\rangle_{U_{h}}+\frac{1}{2}\left\langle\sigma_{y}^{n}\left(u^{n+1}-u^{n}\right), \sigma_{y}^{n}\left(u^{n+1}-u^{n}\right)\right\rangle_{V_{h}} .
\end{aligned}
$$

(vi) Combining the equations (3.32), (3.35) and (3.36), we get

$$
\begin{aligned}
& \left\langle\sigma_{x}^{n+1} u^{n+1}, \sigma_{x}^{n+1} u^{n+1}\right\rangle_{U_{h}}-\left\langle\sigma_{x}^{n} u^{n}, \sigma_{x}^{n} u^{n}\right\rangle_{U_{h}}+\left\langle\sigma_{x}^{n}\left(u^{n+1}-u^{n}\right), \sigma_{x}^{n}\left(u^{n+1}-u^{n}\right)\right\rangle_{U_{h}} \\
& +\left\langle\sigma_{y}^{n+1} v^{n+1}, \sigma_{y}^{n} v^{n+1}\right\rangle_{V_{h}}-\left\langle\sigma_{y}^{n} v^{n}, \sigma_{y}^{n} v^{n}\right\rangle_{V_{h}}+\left\langle\sigma_{y}^{n}\left(v^{n+1}-v^{n}\right), \sigma_{y}^{n}\left(v^{n+1}-v^{n}\right)\right\rangle_{V_{k}} \\
& +\frac{\delta t^{2}}{\chi}\left(\left\langle D_{x} p^{n+1}, D_{x} p^{n+1}\right\rangle_{U_{h}}-\left\langle D_{x} p^{n}, D_{x} p^{n}\right\rangle_{U_{h}}+\left\langle D_{y} p^{n+1}, D_{y} p^{n+1}\right\rangle_{V_{h}}-\left\langle D_{y} p^{n}, D_{y} p^{n}\right\rangle_{V_{h}}\right) \\
& +\frac{\delta t^{2}}{\chi}\left(\left\langle D_{x}\left(p^{n}-p^{n-1}\right), D_{x}\left(p^{n}-p^{n-1}\right)\right\rangle_{U_{h}}+\left\langle D_{y}\left(p^{n}-p^{n-1}\right), D_{y}\left(p^{n}-p^{n-1}\right)\right\rangle_{V_{h}}\right) \\
& +2 \delta t\left\langle\sqrt{\eta^{n}} d_{x} u^{n+1}, \sqrt{\eta^{n}} d_{x} u^{n+1}\right\rangle_{P_{h}}+2 \delta t\left\langle\sqrt{A_{y}\left(A_{x} \eta^{n}\right)} D_{y} u^{n+1}, \sqrt{A_{y}\left(A_{x} \eta^{n}\right)} D_{y} u^{n+1}\right\rangle_{N_{h}} \\
& +2 \delta t\left\langle\sqrt{A_{x}\left(A_{y} \eta^{n}\right)} D_{x} v^{n+1}, \sqrt{A_{x}\left(A_{y} \eta^{n}\right)} D_{y} v^{n+1}\right\rangle_{N_{h}}+2 \delta t\left\langle\sqrt{\eta^{n}} d_{y} v^{n+1}, \sqrt{\eta^{n}} d_{y} v^{n+1}\right\rangle_{P_{n}} \\
& \leq-2 \delta t\left\langle A_{x} \phi^{n} D_{x} w^{n+1}, u^{n+1}\right\rangle_{U_{h}}-2 \delta t\left\langle A_{y} \phi^{n} D_{y} w^{n+1}, v^{n+1}\right\rangle_{V_{h}} \\
& +2 \delta t\left\langle A_{y}\left(A_{x} \eta^{n}\right) D_{y} u^{n+1}, u^{n+1}\right\rangle_{\Gamma_{N S}}+2 \delta t\left\langle A_{x}\left(A_{y} \eta^{n}\right) D_{x} v^{n+1}, v^{n+1}\right\rangle_{\Gamma_{E W}} .
\end{aligned}
$$

(vii) For the boundary term in (3.37), using (3.18), we can derive

$$
\begin{aligned}
& 2 \delta t\left\langle A_{y}\left(A_{x} \eta^{n}\right) D_{y} u^{n+1}, u^{n+1}\right\rangle_{\Gamma_{N S}}+2 \delta t\left\langle A_{x}\left(A_{y} \eta^{n}\right) D_{x} v^{n+1}, v^{n+1}\right\rangle_{\Gamma_{E W}} \\
= & 2 \delta t\left\langle\sigma_{N S}\left(-A_{y}\left(A_{x}\left(\eta^{n} \beta^{n}\right) u^{n+1}\right)+\lambda A_{x} \tilde{L}^{n+1} D_{x}\left(A_{y} \phi^{n}\right)\right), u^{n+1}\right\rangle_{\Gamma_{N S}} \\
& +2 \delta t\left\langle\sigma_{E W}\left(-A_{x}\left(A_{y}\left(\eta^{n} \beta^{n}\right) v^{n+1}\right)+\lambda A_{y} \tilde{L}^{n+1} D_{y}\left(A_{x} \phi^{n}\right)\right), v^{n+1}\right\rangle_{\Gamma_{E W}} \\
= & -2 \delta t\left\langle\sigma_{N S} A_{y}\left(A_{x}\left(\eta^{n} \beta^{n}\right)\right) A_{y} u^{n+1}, u^{n+1}\right\rangle_{\Gamma_{N S}}-2 \delta t\left\langle\sigma_{E W} A_{x}\left(A_{y}\left(\eta^{n} \beta^{n}\right)\right) A_{x} v^{n+1}, v^{n+1}\right\rangle_{\Gamma_{E W}} \\
& \left.+2 \lambda \delta t\left\langle\sigma_{N S} A_{x} \tilde{L}^{n+1} D_{x}\left(A_{y} \phi^{n}\right), u^{n+1}\right\rangle_{\Gamma_{N S}}+2 \lambda \delta t<\sigma_{E W} A_{y} \tilde{L}^{n+1} D_{y}\left(A_{x} \phi^{n}\right), v^{n+1}\right\rangle_{\Gamma_{E W}} .
\end{aligned}
$$

(viii) Taking the discrete inner-product of (3.13) with $2 w^{n+1}$, we have

$$
\begin{aligned}
& 2\left\langle\phi^{n+1}-\phi^{n}, w^{n+1}\right\rangle_{P_{h}}-2 \delta t\left\langle A_{x} \phi^{n} u^{n+1}, D_{x} w^{n+1}\right\rangle_{U_{h}}-2 \delta t\left\langle A_{y} \phi^{n} v^{n+1}, D_{y} w^{n+1}\right\rangle_{V_{h}} \\
& \quad+2 \delta t M\left\langle D_{x} w^{n+1}, D_{x} w^{n+1}\right\rangle_{U_{h}}+2 \delta t M\left\langle D_{y} w^{n+1}, D_{y} w^{n+1}\right\rangle_{V_{h}}=0 .
\end{aligned}
$$


(ix) Taking the discrete inner-product of (3.14) with $-2\left(\phi^{n+1}-\phi^{n}\right)$, we have

$$
\begin{aligned}
& -2\left\langle\phi^{n+1}-\phi^{n}, w^{n+1}\right\rangle_{P_{h}}+\lambda \varepsilon\left(\left\langle D_{x} \phi^{n+1}, D_{x} \phi^{n+1}\right\rangle_{U_{h}}-\left\langle D_{x} \phi^{n}, D_{x} \phi^{n}\right\rangle_{U_{h}}\right. \\
& \left.+\left\langle D_{x}\left(\phi^{n+1}-\phi^{n}\right), D_{x}\left(\phi^{n+1}-\phi^{n}\right)\right\rangle_{U_{h}}\right)+\lambda \varepsilon\left(\left\langle D_{y} \phi^{n+1}, D_{y} \phi^{n+1}\right\rangle_{V_{h}}-\left\langle D_{y} \phi^{n}, D_{y} \phi^{n}\right\rangle_{V_{h}}\right. \\
& \left.+\left\langle D_{y}\left(\phi^{n+1}-\phi^{n}\right), D_{y}\left(\phi^{n+1}-\phi^{n}\right)\right\rangle_{V_{h}}\right) \\
= & 2 \lambda \varepsilon\left\langle D_{y} \phi^{n+1}, \phi^{n+1}-\phi^{n}\right\rangle_{\Gamma_{N S}}+2 \lambda \varepsilon\left\langle D_{x} \phi^{n+1}, \phi^{n+1}-\phi^{n}\right\rangle_{\Gamma_{E W}} \\
& -2 \lambda\left\langle\hat{f}\left(\phi^{n}\right)+S_{1}\left(\phi^{n+1}-\phi^{n}\right), \phi^{n+1}-\phi^{n}\right\rangle_{P_{h}} .
\end{aligned}
$$

(x) For the boundary term in (3.40), applying (3.19) we have,

$$
\begin{aligned}
& 2 \lambda \varepsilon\left\langle D_{y} \phi^{n+1}, \phi^{n+1}-\phi^{n}\right\rangle_{\Gamma_{N S}}+2 \lambda \varepsilon\left\langle D_{x} \phi^{n+1}, \phi^{n+1}-\phi^{n}\right\rangle_{\Gamma_{E W}} \\
= & 2 \lambda\left\langle\sigma_{N S}\left(\tilde{L}^{n+1}-g^{\prime}\left(\phi^{n}\right)-S_{2}\left(\phi^{n+1}-\phi^{n}\right)\right), \phi^{n+1}-\phi^{n}\right\rangle_{\Gamma_{N S}} \\
& +2 \lambda\left\langle\sigma_{E W}\left(\tilde{L}^{n+1}-g^{\prime}\left(\phi^{n}\right)-S_{2}\left(\phi^{n+1}-\phi^{n}\right)\right), \phi^{n+1}-\phi^{n}\right\rangle_{\Gamma_{E W}} \\
= & 2 \lambda \delta t\left\langle\sigma_{N S} \tilde{L}^{n+1},-\gamma \tilde{L}^{n+1}\right\rangle_{\Gamma_{N S}}+2 \lambda \delta t\left\langle\sigma_{E W} \tilde{L}^{n+1},-\gamma \tilde{L}^{n+1}\right\rangle_{\Gamma_{E W}} \\
& -2 \lambda \delta t\left\langle\sigma_{N S} \tilde{L}^{n+1}, A_{x}\left(A_{y} u^{n+1}\right) d_{x}\left(A_{x}\left(A_{y} \phi^{n}\right)\right)\right\rangle_{\Gamma_{N S}} \\
& -2 \lambda \delta t\left\langle\sigma_{E W} \tilde{L}^{n+1}, A_{y}\left(A_{x} v^{n+1}\right) d_{y}\left(A_{y}\left(A_{x} \phi^{n}\right)\right)\right\rangle_{\Gamma_{E W}} \\
& -2 \lambda\left\langle\sigma_{N S}\left(g^{\prime}\left(\phi^{n}\right)+S_{2}\left(\phi^{n+1}-\phi^{n}\right)\right), \phi^{n+1}-\phi^{n}\right\rangle_{\Gamma_{N S}} \\
& -2 \lambda\left\langle\sigma_{E W}\left(g^{\prime}\left(\phi^{n}\right)+S_{2}\left(\phi^{n+1}-\phi^{n}\right)\right), \phi^{n+1}-\phi^{n}\right\rangle_{\Gamma_{E W}} .
\end{aligned}
$$

By Taylor expansion of $F(\phi)$ and $g(\phi)$, we know there exist $\xi$ and $\zeta$ such that

$$
\begin{aligned}
& \hat{F}\left(\phi^{n+1}\right)=\hat{F}\left(\phi^{n}\right)+\hat{f}\left(\phi^{n}\right)\left(\phi^{n+1}-\phi^{n}\right)+\frac{\hat{f}^{\prime}(\xi)}{2}\left(\phi^{n+1}-\phi^{n}\right), \\
& g\left(\phi^{n+1}\right)=g\left(\phi^{n}\right)+g^{\prime}\left(\phi^{n}\right)\left(\phi^{n+1}-\phi^{n}\right)+\frac{g^{\prime \prime}(\zeta)}{2}\left(\phi^{n+1}-\phi^{n}\right) .
\end{aligned}
$$

(xi) Combing equations (3.39) - (3.41) and applying the Taylor expansion, we have

$$
\begin{aligned}
& \lambda \varepsilon\left(\left\langle D_{x} \phi^{n+1}, D_{x} \phi^{n+1}\right\rangle_{U_{h}}-\left\langle D_{x} \phi^{n}, D_{x} \phi^{n}\right\rangle_{U_{h}}+\left\langle D_{x}\left(\phi^{n+1}-\phi^{n}\right), D_{x}\left(\phi^{n+1}-\phi^{n}\right)\right\rangle_{U_{h}}\right) \\
& +\lambda \varepsilon\left(\left\langle D_{y} \phi^{n+1}, D_{y} \phi^{n+1}\right\rangle_{U_{h}}-\left\langle D_{y} \phi^{n}, D_{y} \phi^{n}\right\rangle_{U_{h}}+\left\langle D_{y}\left(\phi^{n+1}-\phi^{n}\right), D_{y}\left(\phi^{n+1}-\phi^{n}\right)\right\rangle_{U_{h}}\right) \\
& -2 \delta t\left\langle A_{x} \phi^{n} u^{n+1}, D_{x} w^{n+1}\right\rangle_{U_{h}}-2 \delta t\left\langle A_{y} \phi^{n} v^{n+1}, D_{y} w^{n+1}\right\rangle_{V_{h}} \\
& +2 \delta t M\left\langle D_{x} w^{n+1}, D_{x} w^{n+1}\right\rangle_{U_{h}}+2 \delta t M\left\langle D_{y} w^{n+1}, D_{y} w^{n+1}\right\rangle_{V_{h}} \\
& =-2 \lambda \delta t\left\langle\sigma_{N S} \tilde{L}^{n+1}, \gamma \tilde{L}^{n+1}\right\rangle_{\Gamma_{N S}}-2 \lambda \delta t\left\langle\sigma_{E W} \tilde{L}^{n+1}, \gamma \tilde{L}^{n+1}\right\rangle_{\Gamma_{E W}} \\
& -2 \lambda \delta t\left\langle\sigma_{N S} \tilde{L}^{n+1}, A_{x}\left(A_{y} u^{n+1}\right) d_{x}\left(A_{x}\left(A_{y} \phi^{n}\right)\right)\right\rangle_{\Gamma_{N S}} \\
& -2 \lambda \delta t\left\langle\sigma_{E W} \tilde{L}^{n+1}, A_{y}\left(A_{x} v^{n+1}\right) d_{y}\left(A_{y}\left(A_{x} \phi^{n}\right)\right)\right\rangle_{\Gamma_{E W}} \\
& -2 \lambda\left\langle\sigma_{N S}\left(g\left(\phi^{n+1}\right)-g\left(\phi^{n}\right)\right), 1\right\rangle_{\Gamma_{N S}}-2 \lambda\left\langle\sigma_{N S}\left(S_{2}-\frac{g^{\prime \prime}(\zeta)}{2}\right),\left(\phi^{n+1}-\phi^{n}\right)^{2}\right\rangle_{\Gamma_{N S}} \\
& -2 \lambda\left\langle\sigma_{E W}\left(g\left(\phi^{n+1}\right)-g\left(\phi^{n}\right)\right), 1\right\rangle_{\Gamma_{E W}}-2 \lambda\left\langle\sigma_{E W}\left(S_{2}-\frac{g^{\prime \prime}(\zeta)}{2}\right),\left(\phi^{n+1}-\phi^{n}\right)^{2}\right\rangle_{\Gamma_{E W}} \\
& -2 \lambda\left\langle\hat{F}\left(\phi^{n+1}\right)-\hat{F}\left(\phi^{n}\right), 1\right\rangle_{P_{n}}-2 \lambda\left\langle S_{1}-\frac{f^{\prime}\left(\phi^{n}\right)}{2},\left(\phi^{n+1}-\phi^{n}\right)^{2}\right\rangle_{P_{n}} .
\end{aligned}
$$


(xii) Finally, summing up equation (3.37), (3.38) and (3.42), we get,

$$
\begin{aligned}
& \frac{1}{2}\left\|\sigma^{n+1} \boldsymbol{u}^{n+1}\right\|_{2}^{2}-\frac{1}{2}\left\|\sigma^{n} \boldsymbol{u}^{n}\right\|_{2}^{2}+\lambda\left(\frac{\varepsilon}{2}\left\|\nabla \phi^{n+1}\right\|_{2}^{2}-\frac{\varepsilon}{2}\left\|\nabla \phi^{n}\right\|_{2}^{2}+\frac{\varepsilon}{2}\left\|\nabla \phi^{n+1}-\nabla \phi^{n}\right\|_{2}^{2}\right) \\
& +\lambda\left(\hat{F}\left(\phi^{n+1}\right)-\hat{F}\left(\phi^{n}\right), 1\right)+\lambda\left(g\left(\phi^{n+1}\right)-g\left(\phi^{n}\right), 1\right)_{\Gamma}+\frac{\delta t^{2}}{2 \chi}\left(\left\|\nabla p^{n+1}\right\|_{2}^{2}-\left\|\nabla p^{n}\right\|_{2}^{2}\right)+\frac{\delta t^{2}}{2 \chi}\left\|\nabla p^{n+1}-\nabla p^{n}\right\|_{2}^{2} \\
\leq & -\delta t\left\|\sqrt{\eta^{n}} \nabla \boldsymbol{u}^{n+1}\right\|_{2}^{2}-\delta t M\left\|\nabla w^{n+1}\right\|_{2}^{2}-\delta t\left\|\sqrt{\eta^{n} \beta^{n}} u_{s}^{n+1}\right\|_{\Gamma}^{2}-\lambda \delta t \gamma\left\|\tilde{L}^{n+1}\right\|_{\Gamma}^{2} \\
& -\lambda h_{x} h_{y}\left\langle S_{1}-\frac{f^{\prime}\left(\phi^{n}\right)}{2},\left(\phi^{n+1}-\phi^{n}\right)^{2}>_{P_{h}}-\lambda h_{x} h_{y}\left\langle\sigma_{N S}\left(S_{2}-\frac{g^{\prime \prime}(\zeta)}{2}\right),\left(\phi^{n+1}-\phi^{n}\right)^{2}\right\rangle_{\Gamma_{N S}}\right. \\
& -\lambda h_{x} h_{y}\left\langle\sigma_{E W}\left(S_{2}-\frac{g^{\prime \prime}(\zeta)}{2}\right),\left(\phi^{n+1}-\phi^{n}\right)^{2}\right\rangle_{\Gamma_{E W}} .
\end{aligned}
$$

Now by the assumption of $S_{1} \geq L_{1} / 2$, and $S_{2} \geq L_{2} / 2$, we can conclude the desired energy stability estimate.

We would like to remark that the unconditionally energy stable scheme allows us to use large time steps. Nevertheless, large time steps may produce non-physical solutions. For the sake of numerical accuracy, we adjust time steps (cf. [35]) based on the total energy derivative as follows

$$
\Delta t=\max \left(\Delta t_{\min }, \frac{\Delta t_{\max }}{\sqrt{1+\alpha\left|E^{\prime}(t)\right|^{2}}}\right),
$$

where $E(t)$ is the system energy, $\alpha$ is an adjustable constant, $\Delta t_{\min }$ and $\Delta t_{\max }$ are pre-set lower and upper bound for the time step respectively. The large deviation in energy will lead to a small time step, which corresponds to the case of rapid decay of energy. Similarly, the small change in energy will yield a large time step.

\section{Numerical Results}

In this section, numerical experiments are conducted to validate accuracy, efficiency and energy decay property of the proposed scheme. We first use a classical case to perform a convergence test, and then present a 2-D bubble rising case and a 3-D droplet spreading on a chemical patterned surface.

\subsection{Two-phase Couette flow}

The two-phase Couette flow with different densities and viscosity is conducted to test the convergence of numerical schemes. To avoid the possible error introduced by the adaptive time step, a constant time step will be used in this section. The initial value of $\phi$ is set as

$$
\phi(x, y)=\tanh \left(\frac{1}{\sqrt{2} \varepsilon}\left(\frac{L_{x}}{4}-\left|x-\frac{L_{x}}{2}\right|\right)\right) .
$$

The dynamic contact line boundary condition is used for the phase variable and the GNBC is used for the velocity at the south and north walls while periodic boundary conditions are imposed on the west and east boundaries. The south and north walls are moving with $u_{w}=0.2$ in opposite directions. The parameters used in the simulation are listed as follows:

$$
\begin{aligned}
& L_{x}=3, \quad L_{y}=1, \quad \rho_{1}=1, \quad \rho_{2}=1.1, \quad \eta_{1}=1, \quad \eta_{2}=1.1, \quad M=1 \times 10^{-3}, \quad \varepsilon=0.01, \quad \lambda=1.2 \\
& \gamma=100, \quad \theta_{\mathrm{s}}=60^{\circ}, \quad \eta \beta=1 / 0.19 .
\end{aligned}
$$


We first use a constant time step $\delta t=0.001$ and a spatial resolution $n_{x}=300, n_{y}=100$ to solve the coupled system. Figure 1 gives the results at $t=2$.

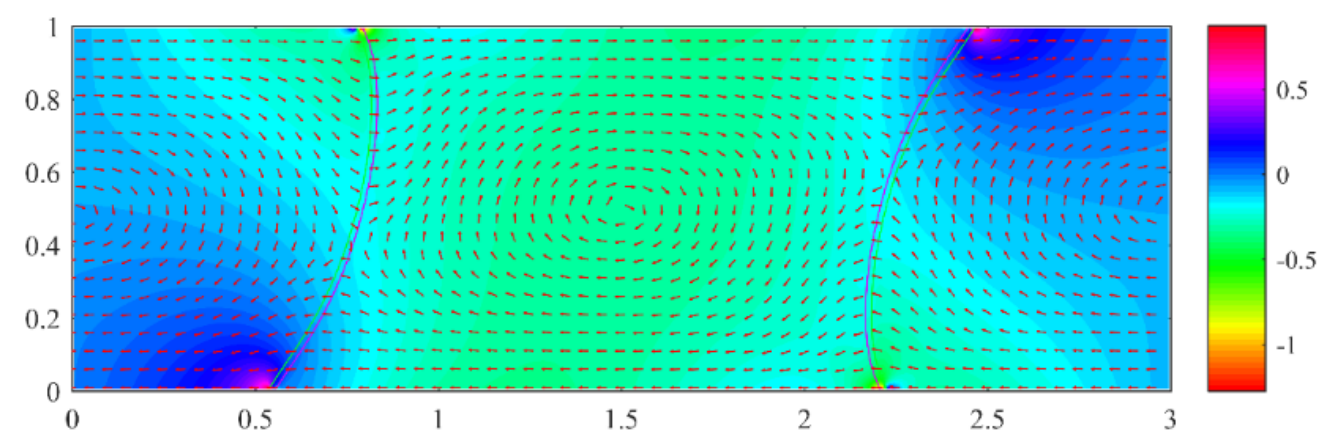

Figure 1. The pressure field (background color), contour line of $\phi=(-0.5,0.5)$ and the quiver plot of velocity $u, v$ at $t=2$.

To test the convergence of time step $\delta t$, a spatial resolution $n_{x}=450, n_{y}=150$ is used to reduce the error related to the spatial discretization. The solution obtained with a small time step $\delta t$ $=6.25 \times 10^{-5}$ is used as an exact solution due to the absence of exact solution. Different time steps $\delta t$ $=1 \times 10^{-3}, 5 \times 10^{-4}, 2.5 \times 10^{-4}, 1.25 \times 10^{-4}$ are selected to calculate the $L^{2}$ norm error and convergence order for the phase variable $\phi$, the velocity $u$ and the pressure $p$ at $t=0.1$. The temporal convergence properties are presented in Table 1 . We can conclude that the proposed numerical scheme can converge by the first order accuracy in time.

Table 1. The $L^{2}$ norm error and convergence order for the phase variable $\phi$, the velocity $u$ and the pressure $p$ at $t=$ 0.1 with different temporal resolutions.

\begin{tabular}{|c|c|c|c|c|c|c|}
\hline$\delta t$ & Error $(\phi)$ & Order & Error $(u)$ & Order & Error $(p)$ & Order \\
\hline $1.00 \mathrm{E}-03$ & $3.96 \mathrm{E}-02$ & - & $3.30 \mathrm{E}-02$ & - & $8.56 \mathrm{E}-02$ & - \\
\hline $5.00 \mathrm{E}-04$ & $2.22 \mathrm{E}-02$ & 0.83 & $1.92 \mathrm{E}-02$ & 0.78 & $5.93 \mathrm{E}-02$ & 0.53 \\
\hline $2.50 \mathrm{E}-04$ & $9.71 \mathrm{E}-03$ & 1.20 & $8.47 \mathrm{E}-03$ & 1.18 & $3.12 \mathrm{E}-02$ & 0.93 \\
\hline $1.25 \mathrm{E}-04$ & $2.75 \mathrm{E}-03$ & 1.79 & $1.55 \mathrm{E}-03$ & 1.70 & $9.08 \mathrm{E}-03$ & 1.78 \\
\hline
\end{tabular}

To further test the spatial convergence in $x$, we use $n_{y}=100$ and $\delta t=0.1 h_{x}$ for a series of $n_{x}=$ $200,400,600,800$. The solution at $n_{x}=1000$ is used as a reference solution. The $L^{2}$ norm error for the phase variable, the velocity and the pressure at $t=2$ is calculated. The system at $t=2$ is close to a steady state, so the error related to the temporal discretization can be neglected. Similarly, we use $n_{x}=300$ and $\delta t=0.1 h_{y}$ for a series of $n_{y}=80,120,160,200$ to test the spatial convergence in $y$. The exact solution is obtained with $n_{y}=1000, \delta t=0.1 h_{y}$. Again, the $L^{2}$ norm error is calculated at $t=2$. We can conclude from Figure 2 that the proposed scheme deserves the first order accuracy in space. 

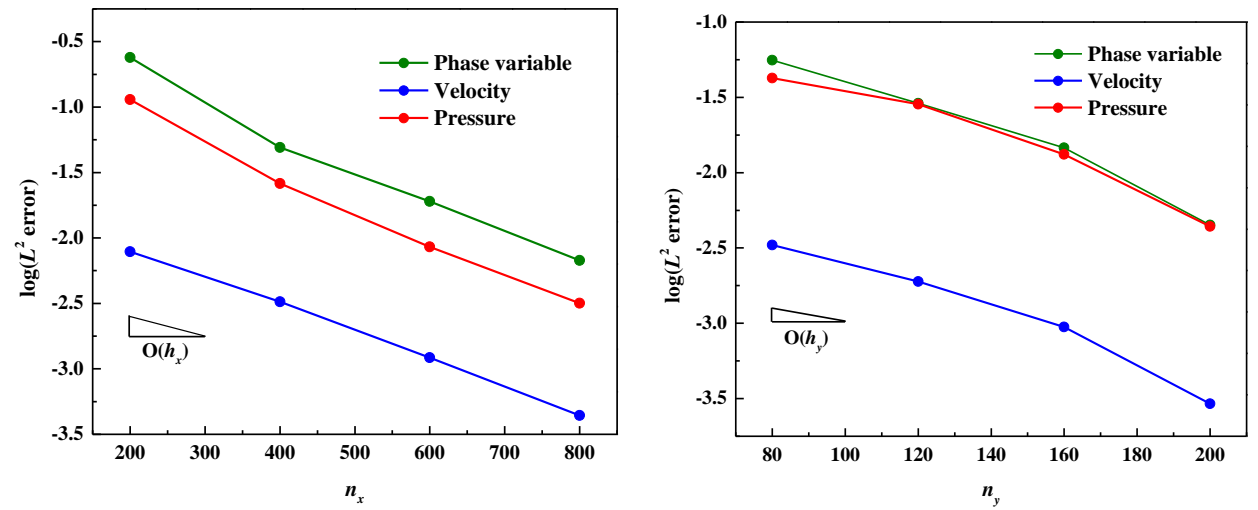

Figure 2. $L^{2}$ norm error for the phase variable $\phi$, the velocity $u$ and the pressure $p$ at $t=2$ with different spatial resolutions.

Now, we verify the discrete energy law of the numerical scheme based on the finite difference method on the staggered grids. To ensure no output energy, we set the velocity of wall $u_{w}=0$. The spatial resolution is $n_{x}=300, n_{y}=100$. Figure 3 gives the energy evolution with three different time steps. The tendency of energy curves confirms the decreasing property of the discrete energy. In addition, there are obvious differences between large time steps and small time steps, which indicate that large time steps induce more numerical errors.

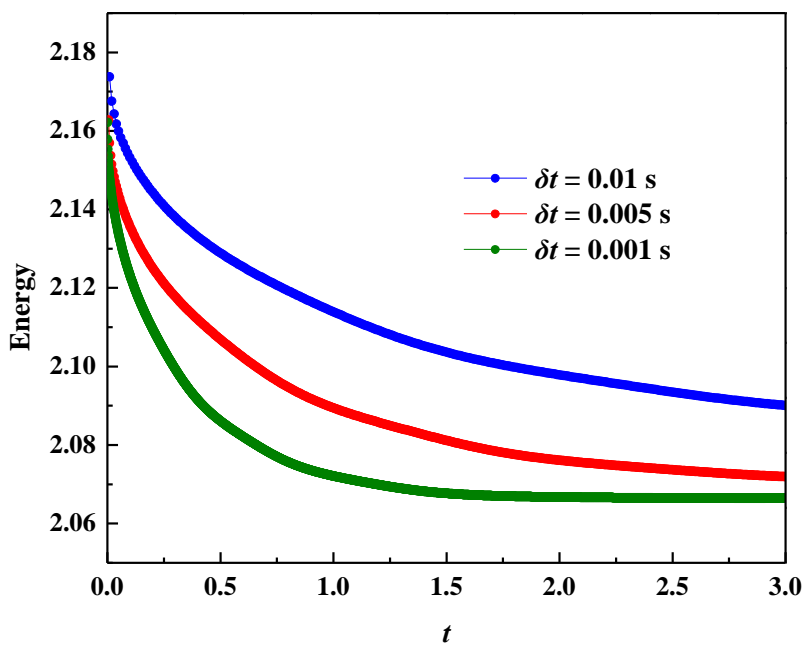

Figure 3. Energy dissipation properties with different time steps.

\subsection{Bubble Rising Process}

In this section, we simulate a lighter bubble rising process in a rectangular domain $\Omega=[0,1]$ $\times[0,2]$. The dynamic contact line boundary condition and the GNBC are used for all walls. The initial profile of $\phi$ is set as

$$
\phi(x, y)=-\tanh \left(\frac{1}{\sqrt{2} \varepsilon}\left(\sqrt{\left(x-x_{0}\right)^{2}+\left(y-y_{0}\right)^{2}}-r\right)\right) .
$$

Parameters used in simulations are as follows:

$$
\begin{aligned}
& \rho_{1}=100, \quad \rho_{2}=1000, \quad \eta_{1}=1, \quad \eta_{2}=10, \quad M=1 \times 10^{-5}, \quad \varepsilon=0.008, \quad \lambda=25.99, \quad \gamma=500, \\
& \theta_{\mathrm{s}}=60^{\circ}, \quad \eta \beta=1 / 0.19, \quad n_{x}=180, \quad n_{y}=360, \quad x_{0}=0.5, \quad y_{0}=0.5, \quad r=0.25 .
\end{aligned}
$$

An adaptive time strategy is used in simulations. Although a scheme with unconditional energy stability can allow arbitrarily large time steps, a larger time step will definitely induce 
larger numerical error. In the time adaptive simulations, the maximum time step is limited to maintain the desirable accuracy. We set the minimum time step $\delta t_{\min }=0.1 h_{x}$, the maximum time step $\delta t_{\max }=0.5 h_{x}$ and the adjustable constant $\alpha=100$, respectively. The shape evolution of the rising bubble is shown in Figure 4. We notice that the two sides of bubble touch the top wall firstly in Figure 4 (d). We remark that in other cases, the central part of bubble may touch the top wall firstly if the size of bubble increases.

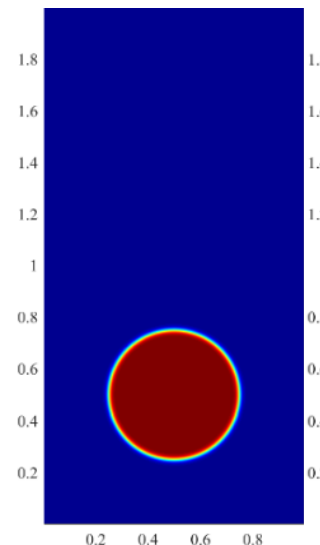

(a) $0 \mathrm{~s}$

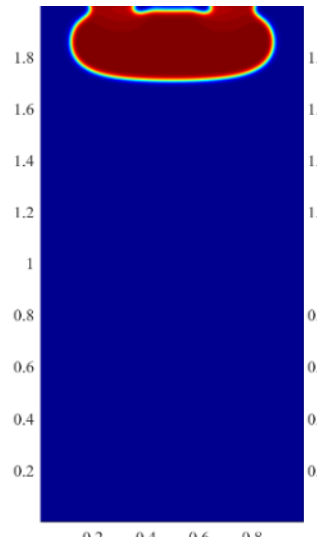

(e) $7.572 \mathrm{~s}$

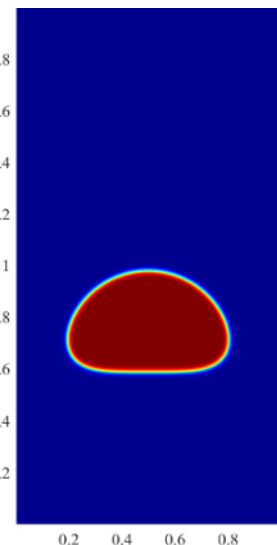

(b) $1.5 \mathrm{~s}$

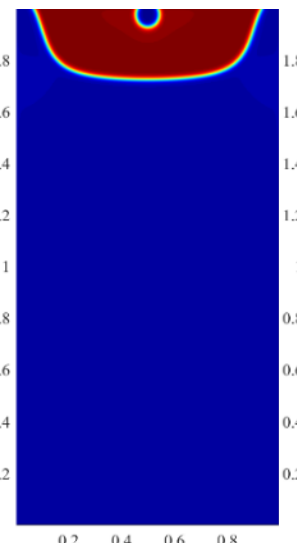

(f) $7.71 \mathrm{~s}$

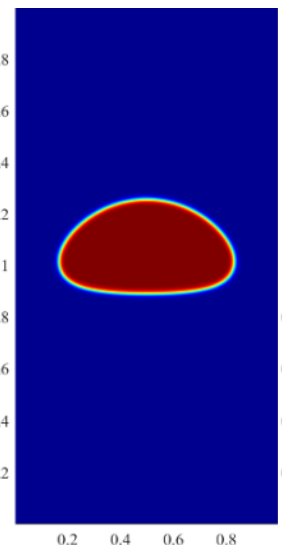

(c) $3 \mathrm{~s}$

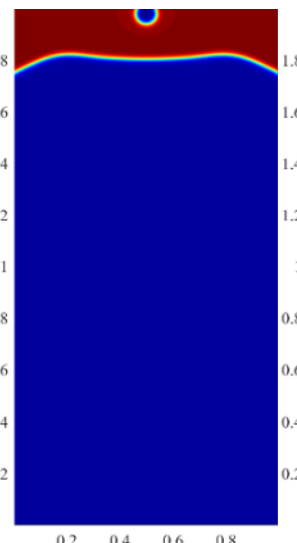

(g) $8.231 \mathrm{~s}$

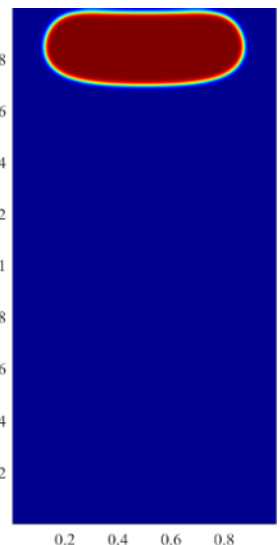

(d) $7.484 \mathrm{~s}$

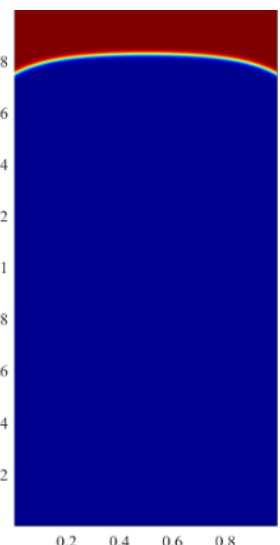

(h) $15 \mathrm{~s}$

Figure 4. The bubble rising dynamics with the contact angle $\theta_{\mathrm{s}}=60^{\circ}$.

Figure 4 (h) gives the final steady state of the lighter phase. To accurately estimate the dynamics of bubble rising process, two measured quantities [36] are introduced, namely, the mass center and mean rise velocity, and they can be calculated by the following formulae:

$$
\text { center of mass }=\frac{\int_{\phi \geq 0} y \rho d \Omega}{\int_{\phi \geq 0} \rho d \Omega}, \quad \text { rise velocity }=\frac{\int_{\phi \geq 0} v d \Omega}{\int_{\phi \geq 0} 1 d \Omega} .
$$




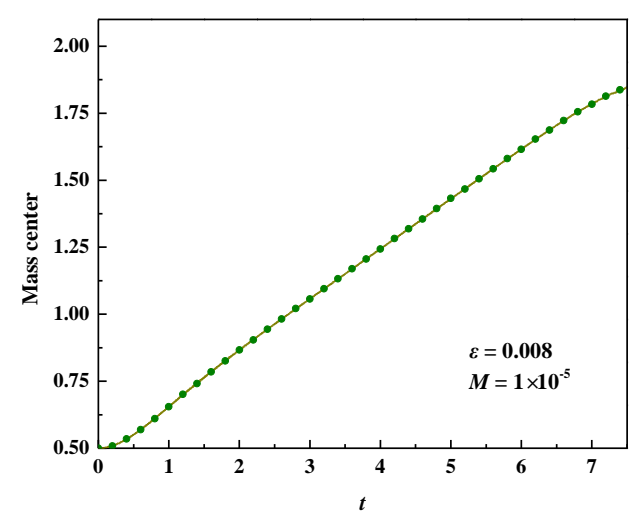

(a)

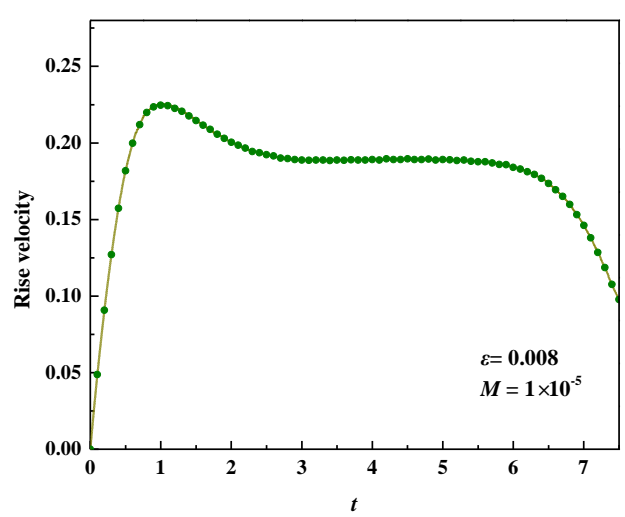

(b)

Figure 5. The mass center and mean rise velocity of the lighter bubble before it touches the top wall with $\varepsilon=0.008$ and $M=1 \times 10^{-5}$. (a) the mass center; (b) the mean rise velocity.

Figure 5 gives the mass center and rise velocity of the lighter bubble before it reaches the top wall. A significant increase in rise velocity can be observed at the initial stage and then the velocity decrease slowly to a constant value at $t=3$. The shape of bubble will reach a temporary steady state when the rise velocity keeps constant. Before the bubble reaches the top wall, the rise velocity sees a dramatic drop.

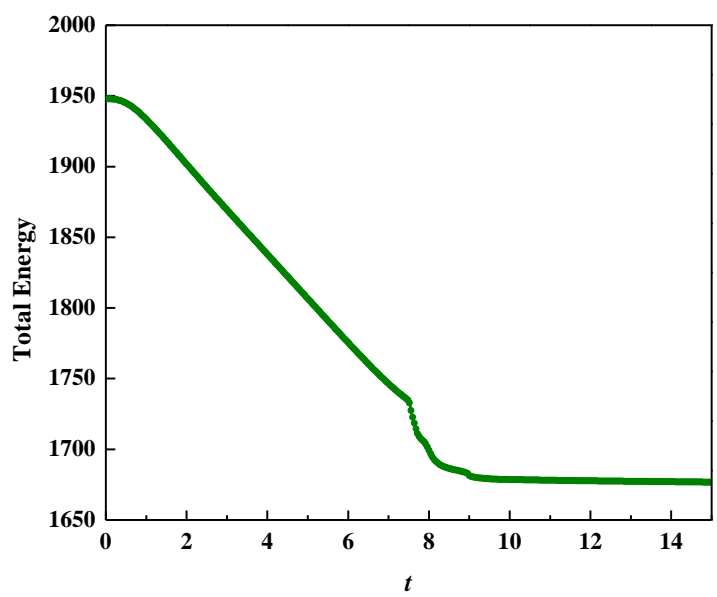

Figure 6. The energy dissipation of the whole system

The decreasing trend of discrete energy in Figure 6 confirms that the proposed scheme is energy stable. The whole system reaches the equilibrium state at around $t=10$. Figure 7 gives the evolutions of three different kinds of energies. At the early stage of bubble rising, the kinetic energy and free energy rise dramatically, which come from part of the reduced gravity potential energy. At around $t=3$, the kinetic energy and free energy remain in balance until $t=6$, and the consumed gravity potential energy is mainly used to deal with heat loss. After that, the kinetic energy drops significantly and transfers into the free energy as the bubble moves to the top wall. The surface energy at fluid-solid boundaries remains the same before the bubble touches the solid wall. To demonstrate the complex energy changes after the bubble touches the north wall, we give the evolutions of energies within the specific time interval ( $t$ from 6 to 10) and mark three important moments in Figure 8. At the moment that bubble touches the north wall, the surface 
energy and free energy decay fast and part of them transfer into the kinetic energy (the first blue dotted line). The kinetic energy has a second increase when the lighter phase touches the west and east walls (the middle blue dotted line). About $t=9$, the blue phase has almost diffused into the red phase completely and the moving contact line on the north wall disappears. Then the kinetic energy and free energy decrease slowly while the surface energy increases slightly until the interface reaches the steady state with the prescribed contact angle.

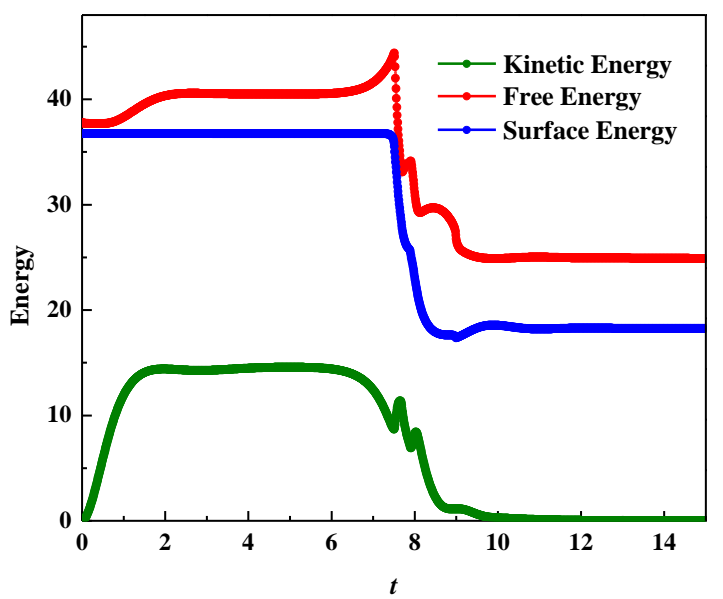

Figure 7. Evolution of kinetic energy, free energy and surface energy.

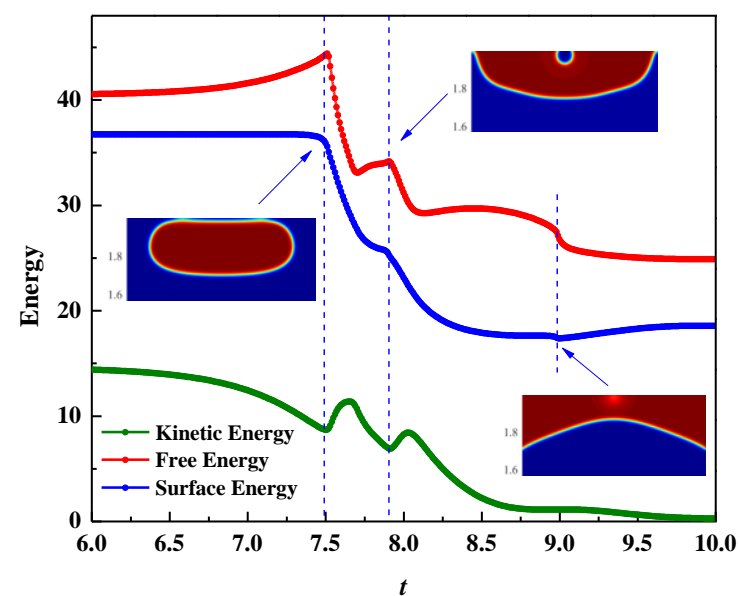

Figure 8. The evolutions of kinetic energy, free energy and surface energy within the specific time interval ( $t$ from 6 to 10$)$.

\subsection{Droplet spreading on a chemically patterned surface}

In this section, 3-D simulations of droplet spreading on a chemically patterned surface [37] are conducted to demonstrate the numerical efficiency of our method. The domain is $\Omega=[0,0.8]^{2}$ $\times[0,0.4]$. The dynamic contact line boundary condition and the GNBC are imposed on the bottom and top walls. The droplet locates at the center of bottom wall with the radius $r=0.25$. On the bottom wall, we set wetting angle $\theta_{1}$ for the subdomain $\Omega_{1}=\left\{(x, y, 0) \in \mathrm{R}^{3}, 0.3 \leq x \leq 0.5,0.3 \leq y \leq 0.5\right\}$ and $\theta_{2}$ for anywhere else. The droplet mainly locates on $\Omega_{1}$. If not explicit specified, the model parameters take default values as follows:

$$
\begin{aligned}
& \rho_{1}=0.9, \quad \rho_{2}=1, \quad \eta_{1}=1.1, \quad \eta_{2}=1, \quad M=1 \times 10^{-3}, \quad \varepsilon=0.012, \\
& \lambda=1.2, \quad \gamma=500, \quad \eta \beta=1 / 0.19, \quad h_{x}=h_{y}=h_{z}=0.01 .
\end{aligned}
$$

The fluid 1 (droplet) has a slightly smaller density and larger viscosity, can be regarded as oil 
phase. Similarly, the fluid 2 with a slightly larger density and smaller viscosity, can be regarded as water phase. To clearly demonstrate the effect of a patterned surface, a chemically homogeneous surface with the contact angle $\theta_{s}$ is set as a comparison. We first set $\theta_{1}=\theta_{s}=60^{\circ}$ and $\theta_{2}=120^{\circ}$, and simulations are run until the droplet reaches the steady state.

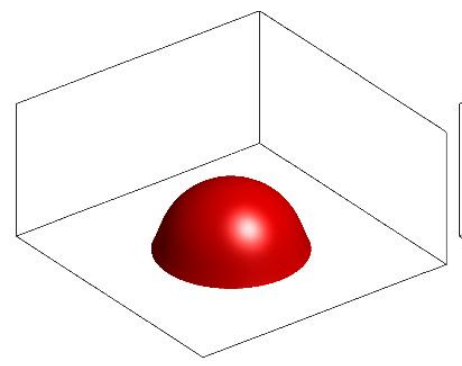

(a) $t=0.04$

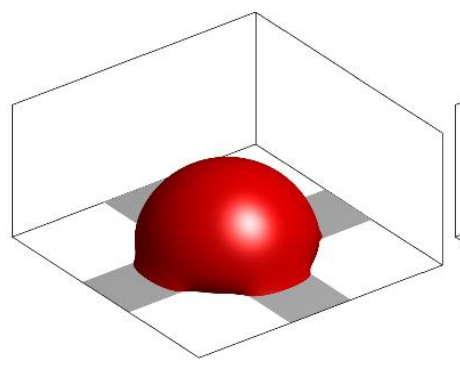

(b) $t=0.04$

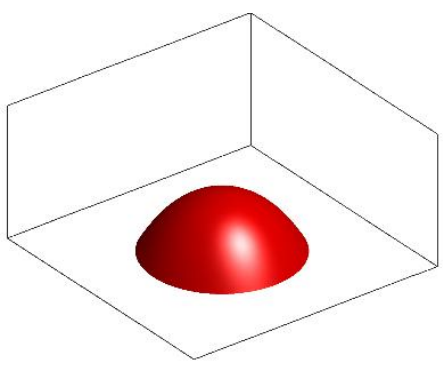

(a) $t=0.6$

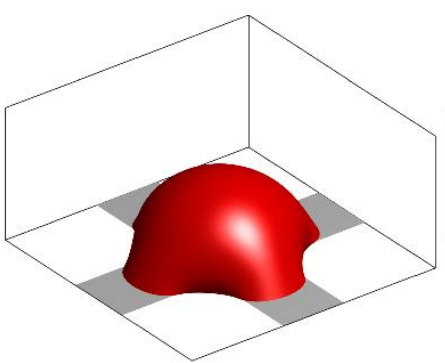

(b) $t=0.6$

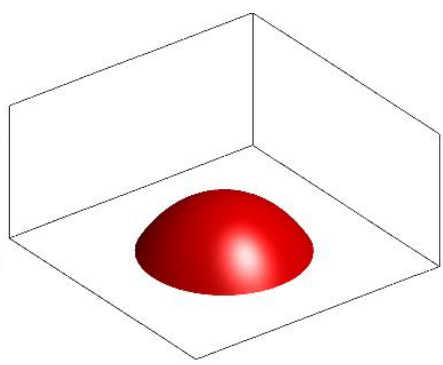

(a) $t=1$

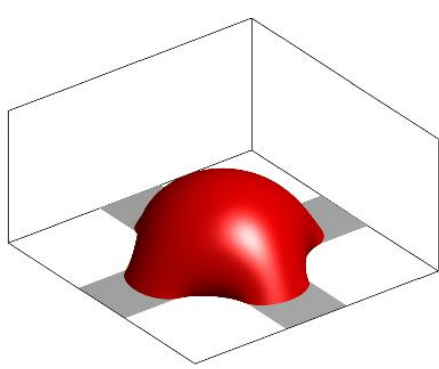

(b) $t=1$

Figure 9. The evolutions of droplets on physically smooth surfaces (a) a chemically homogeneous surface with $\theta_{s}$ $=60^{\circ} ;(\mathrm{b})$ a chemically patterned surface with $\theta_{1}=60^{\circ}$ and $\theta_{2}=120^{\circ}$.

Figure 9 gives the evolutions of droplets on physically smooth surfaces. At $t=1$, the equilibrium of oil droplets is reached on both surfaces. On a chemically patterned surface, the oil droplet contracts inward on a hydrophilic zone and spreads quickly on a hydrophobic zone until the final steady state reaches. The curve in Figure 10 indicates the energy stability of our method. The energy of a homogeneous surface is higher than that of a patterned surface.

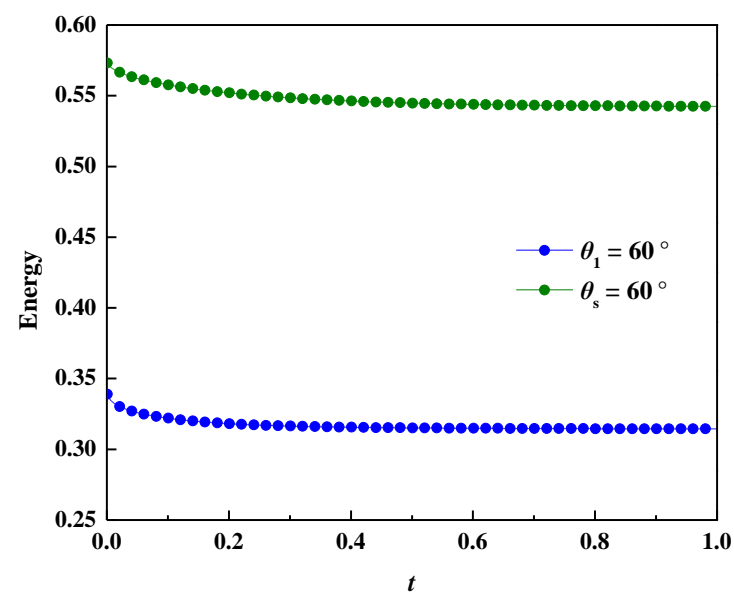

Figure 10. The energy decay curve of the whole system with $\theta_{1}=\theta_{s}=60^{\circ}$ and $\theta_{2}=120^{\circ}$.

Similarly, we set $\theta_{1}=\theta_{s}=120^{\circ}$ and $\theta_{2}=60^{\circ}$, and simulations are run until the droplet reaches the 
steady state.

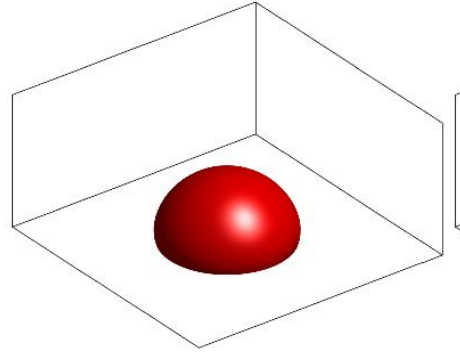

(a) $t=0.04$

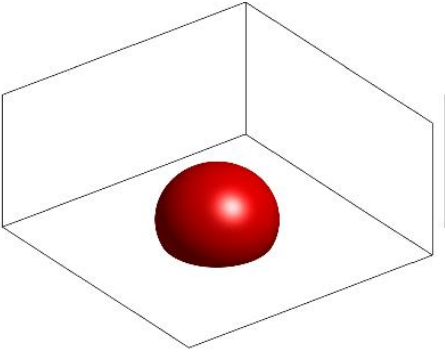

(a) $t=0.6$

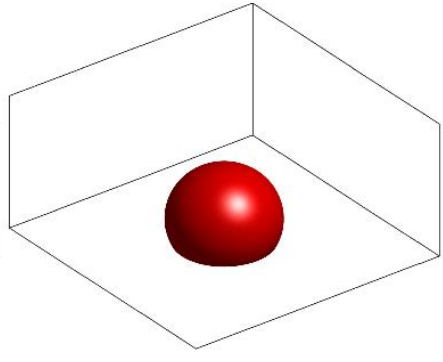

(a) $t=1$

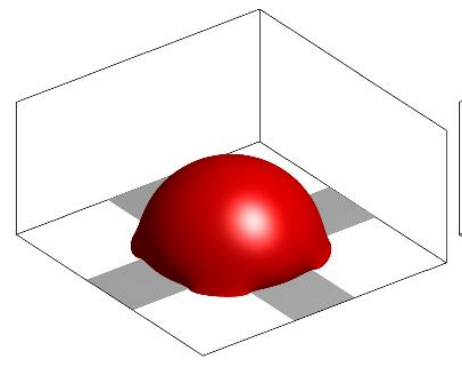

(b) $t=0.04$

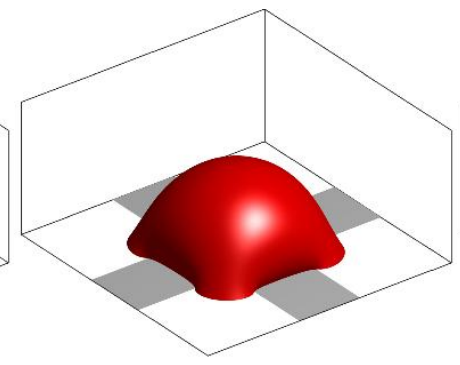

(b) $t=0.6$

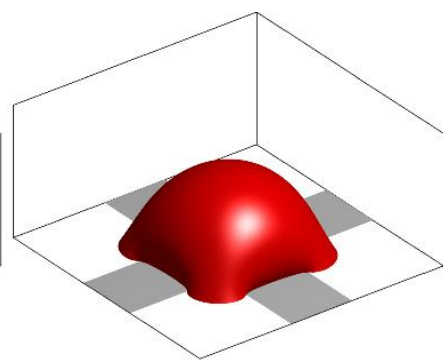

(b) $t=1$

Figure 11. The evolutions of droplets on physically smooth surfaces. (a) a chemically homogeneous surface with $\theta_{s}=120^{\circ}$; (b) a chemically patterned surface with $\theta_{1}=120^{\circ}$ and $\theta_{2}=60^{\circ}$.

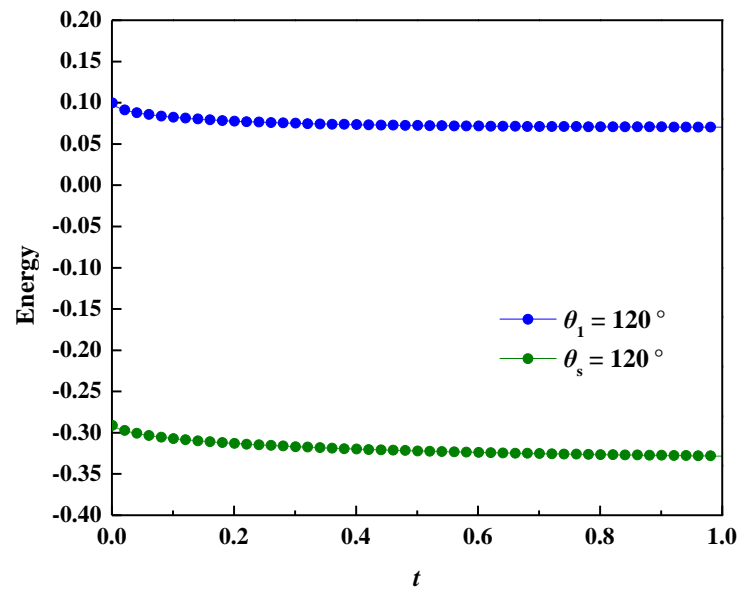

Figure 12. The energy decay curve of the whole system with $\theta_{1}=\theta_{s}=120^{\circ}$ and $\theta_{2}=60^{\circ}$.

As expected, the oil droplet contracts inward on a hydrophilic zone and spreads quickly on a hydrophobic zone and eventually reaches equilibrium on a chemically patterned surface.

Further, we can extend our simulations to a more complicated chemically patterned surface (cf. e.g. [38]), as shown in Figure 13. The domain is $\Omega=(0,0.9)^{2} \times(0,0.5)$. A semi-circular oil droplet is placed on the center of the surface. The radius of oil droplet is 0.28 . The wetting angles for oil droplet of the gray zone and white zone are $\theta_{1}=60^{\circ}$ and $\theta_{2}=120^{\circ}$, respectively. Each square box has the same size. The evolution of the oil droplet is shown in Figure 13. 


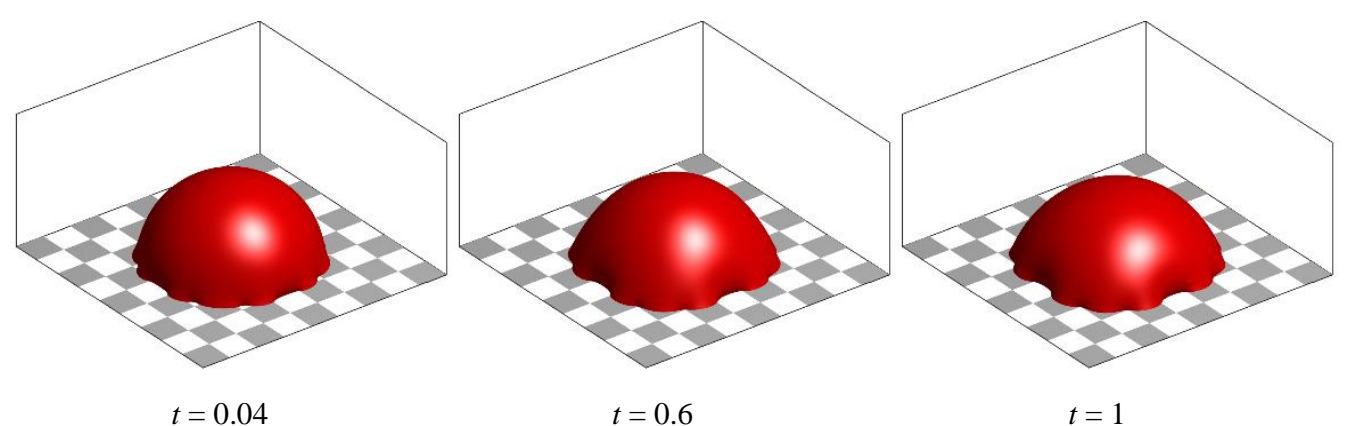

Figure 13. The evolution of droplet on a chemically patterned surface with $\theta_{1}=60^{\circ}$ and $\theta_{2}=120^{\circ}$.

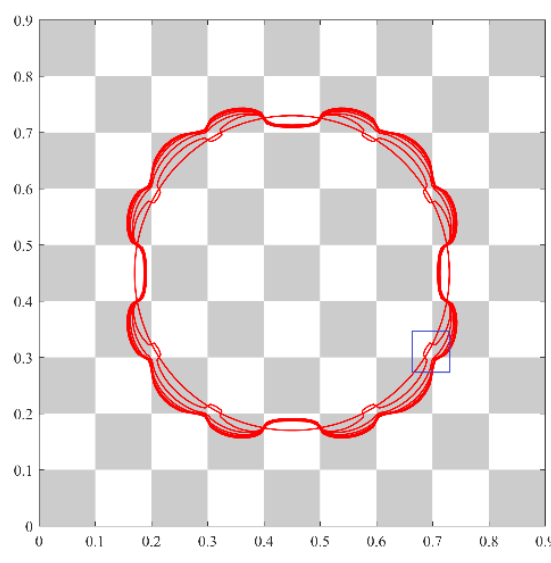

(a)

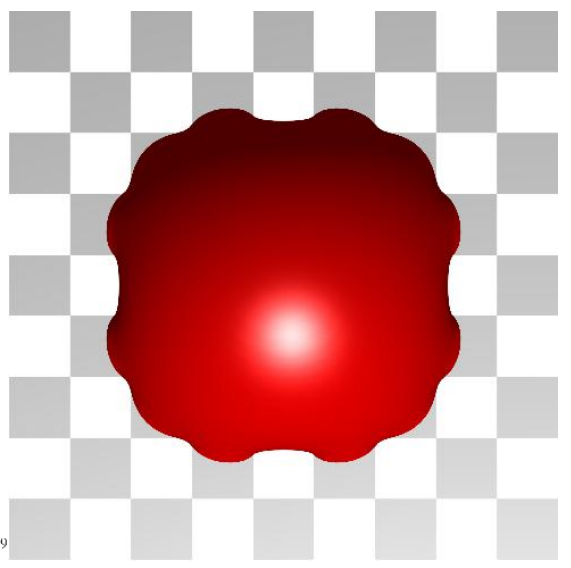

(b)

Figure 14. The evolution of contact line from $t=0$ to $t=1$ and top view of oil droplet at $t=1$. (a) the evolution of contact line; (b) top view of oil droplet at the equilibrium.

Figure 14 gives the positions of contact line from $t=0$ to $t=1$. In general, the oil droplet contracts inward on a hydrophilic zone and spreads quickly on a hydrophobic zone. We can also observe an interesting phenomenon on the corner of two white square boxes, which is marked with a blue rectangle in Figure 14(a). Initially, the oil droplet at the corner contracts inward due to the hydrophilic of white box. Then the oil on the white box moves forward due to the spreading of oil on the gray boxes near the white box, and the oil reaches the corner of two white boxes when the whole system reaches a steady state. The energy decay curve in Figure 15 indicates the stability of the proposed scheme again.

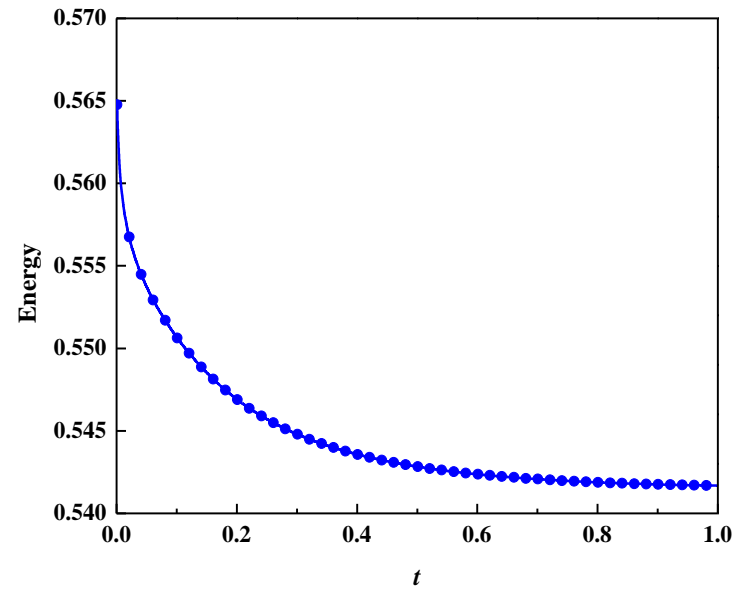

Figure 15. The energy decay curve of the whole system with $\theta_{1}=\theta_{s}=60^{\circ}$ and $\theta_{2}=120^{\circ}$. 


\section{Concluding remarks}

In this work, a fully discrete scheme is presented for a coupled hydrodynamics phase filed model that incorporates moving contact line boundary condition, and variable densities, viscosities. We provide a rigorous proof of energy stability for the fully discrete scheme based on a semi-implicit temporal discretization and a finite difference method on the staggered grids for the spatial discretization. A splitting method based on the pressure stabilization is implemented to solve the Navier-Stokes equation, while a stabilization approach is used for the Cahn-Hilliard equation. The numerical results in both 2-D and 3-D demonstrate the accuracy, efficiency and decaying property of discrete energy of the proposed scheme.

\section{Acknowledgement}

Jun Yao and Guangpu Zhu acknowledge that this work is supported by the National Science and Technology Major Project (2016ZX05011-001), the NSF of China (51490654, 51504276, and 51304232) and the Innovative Project of China University of Petroleum (YCX2017021). The work of Huangxin Chen is supported by the NSF of China (Grant No. 11771363, 91630204, 51661135011) and Program for Prominent Young Talents in Fujian Province University. Shuyu Sun acknowledges that this work is supported by the KAUST research fund awarded to the Computational Transport Phenomena Laboratory at KAUST through the Grant BAS/1/1351-01-01.

\section{References}

[1] N.R. Morrow, Wettability and its effect on oil recovery, J. Petrol. Technol., 42 (1990) 1476-1484.

[2] K. Bao, Y. Shi, S. Sun, and X.-P. Wang, A finite element method for the numerical solution of the coupled Cahn-Hilliard and Navier-Stokes system for moving contact line problems, J. Comput. Phys., 231 (2012) 8083-8099.

[3] E. Dussan, On the spreading of liquids on solid surfaces: static and dynamic contact lines, Annu. Rev. Fluid Mech., 11 (1979) 371-400.

[4] T. Qian, X.-P. Wang, and P. Sheng, Molecular scale contact line hydrodynamics of immiscible flows, Phys. Rev. E, 68 (2003) 016306.

[5] T. Qian, X.-P. Wang, and P. Sheng, Power-law slip profile of the moving contact line in two-phase immiscible flows, Phys. Rev. Lett., 93 (2004) 094501.

[6] G. Tryggvason, B. Bunner, A. Esmaeeli, D. Juric, N. Al-Rawahi, W. Tauber, J. Han, S. Nas, and Y.-J. Jan, A front-tracking method for the computations of multiphase flow, J. Comput. Phys., 169 (2001) 708-759.

[7] D. Jacqmin, Calculation of two-phase Navier-Stokes flows using phase-field modeling, J. Comput. Phys., 155 (1999) 96-127.

[8] X. Yang, J.J. Feng, C. Liu, and J. Shen, Numerical simulations of jet pinching-off and drop formation using an energetic variational phase-field method, J. Comput. Phys., 218 (2006) 417-428.

[9] X. Yang and D. Han, Linearly first-and second-order, unconditionally energy stable schemes for the phase field crystal model, J. Comput. Phys., 330 (2017) 1116-1134.

[10] J. Shen and X. Yang, A phase-field model and its numerical approximation for two-phase incompressible flows with different densities and viscosities, SIAM. J. Sci. Comput., 32 (2010) 1159-1179.

[11] J. Shen and X. Yang, Energy stable schemes for Cahn-Hilliard phase-field model of two-phase incompressible flows, Chinese Annals of Mathematics, Series B, 31 (2010) 743-758. 
[12] J. Shen and X. Yang, Numerical approximations of allen-cahn and cahn-hilliard equations, Discrete Contin. Dyn. Syst., 28 (2010) 1669-1691.

[13] J. Shen and X. Yang, Decoupled, energy stable schemes for phase-field models of two-phase incompressible flows, SIAM J. Numer. Anal., 53 (2015) 279-296.

[14] J. Shen, X. Yang, and H. Yu, Efficient energy stable numerical schemes for a phase field moving contact line model, J. Comput. Phys., 284 (2015) 617-630.

[15] Y. Chen and J. Shen, Efficient, adaptive energy stable schemes for the incompressible Cahn-Hilliard Navier-Stokes phase-field models, J. Comput. Phys., 308 (2016) 40-56.

[16] Y. Jiang, P. Lin, Z. Guo, and S. Dong, Numerical simulation for moving contact line with continuous finite element schemes, Commun. Comput. Phys., 18 (2015) 180-202.

[17] T. Qian, X.-P. Wang, and P. Sheng, Generalized Navier boundary condition for the moving contact line, Commun. Math. Sci., 1 (2003) 333-341.

[18] Q. He, R. Glowinski, and X.-P. Wang, A least-squares/finite element method for the numerical solution of the Navier-Stokes-Cahn-Hilliard system modeling the motion of the contact line, J. Comput. Phys., 230 (2011) 4991-5009.

[19] M. Gao and X.-P. Wang, A gradient stable scheme for a phase field model for the moving contact line problem, J. Comput. Phys., 231 (2012) 1372-1386.

[20] M. Gao and X.-P. Wang, An efficient scheme for a phase field model for the moving contact line problem with variable density and viscosity, J. Comput. Phys., 272 (2014) 704-718.

[21] S. Aland and F. Chen, An efficient and energy stable scheme for a phase - field model for the moving contact line problem, Int. J. Numer. Methods Fluids, 81 (2016) 657-671.

[22] H. Yu and X. Yang, Numerical approximations for a phase-field moving contact line model with variable densities and viscosities, J. Comput. Phys., 334 (2017) 665-686.

[23] R. Chen, X. Yang, and H. Zhang, Decoupled, Energy Stable Scheme for Hydrodynamic Allen-Cahn Phase Field Moving Contact Line Model, arXiv preprint arXiv:1703.06780, (2017).

[24] X. Yang and H. Yu, Linear, Second order and Unconditionally Energy Stable schemes for a phase-field moving contact line Model, arXiv preprint arXiv:1703.01311, (2017).

[25] F.H. Harlow and J.E. Welch, Numerical calculation of time - dependent viscous incompressible flow of fluid with free surface, The physics of fluids, 8 (1965) 2182-2189.

[26] V. L. Lebedev, Difference analogues of orthogonal decompositions, fundamental differential operators and certain boundary-value problems of mathematical physics, Z. Vycisl. Mat. i Mat. Fiz., 4 (1964) 449-465.

[27] J. Li and S. Sun, The superconvergence phenomenon and proof of the MAC scheme for the Stokes equations on non-uniform rectangular meshes, J. Sci. Comput. , 65 (2015) 341-362.

[28] H. Chen, S. Sun, and T. Zhang, Energy stability analysis of some fully discrete numerical schemes for incompressible Navier-Stokes equations on staggered grids, J. Sci. Comput., accepted, 2017.

[29] B.J. Daly, F.H. Harlow, J.P. Shannon, and J.E. Welch, The MAC Method, Tech. report LA-3425, Los Alamos Scientific Laboratory, University of California, 1965.

[30] P. Yue, J.J. Feng, C. Liu, and J. Shen, A diffuse-interface method for simulating two-phase flows of complex fluids, J. Fluid Mech., 515 (2004) 293-317.

[31] L.A. Caffarelli, and N.E. Muler, An $\mathrm{L}^{\infty}$ bound for solutions of the Cahn-Hilliard equation, Arch. Ration. Mech. Anal., 133 (1995) 129-144.

[32] N. Condette, C. Melcher, and E. Süli, Spectral approximation of pattern-forming nonlinear evolution equations with double-well potentials of quadratic growth, Math. Comp., 80 (2011) 205-223. 
[33] J.-L. Guermond, and A. Salgado, A splitting method for incompressible flows with variable density based on a pressure Poisson equation, J. Comput. Phys., 228 (2009) 2834-2846.

[34] S. Wise, Unconditionally stable finite difference, nonlinear multigrid simulation of the Cahn-Hilliard-Hele-Shaw system of equations, J. Sci. Comput. , 44 (2010) 38-68.

[35] Z. Zhang, Y. Ma, and Z. Qiao, An adaptive time-stepping strategy for solving the phase field crystal model, J. Comput. Phys., 249 (2013) 204-215.

[36] S. Aland and A. Voigt, Benchmark computations of diffuse interface models for two - dimensional bubble dynamics, Int. J. Numer. Methods Fluids, 69 (2012) 747-761.

[37] X.-P. Wang, T. Qian, and P. Sheng, Moving contact line on chemically patterned surfaces, J. Fluid Mech., 605 (2008) 59-78.

[38] H. Zhong, X.-P. Wang, and S. Sun, A numerical study of three-dimensional droplets spreading on chemically patterned surfaces, Discrete Contin. Dyn. Syst. Ser. B, 21 (2016) 2905-2926. 